\title{
Mixed Initial-Boundary Value Problem for the Capillary Wave Equation
}

\author{
B. Juarez Campos, ${ }^{1}$ Elena Kaikina, ${ }^{2}$ and Hector F. Ruiz Paredes ${ }^{1}$ \\ ${ }^{1}$ Instituto Tecnologico de Morelia, Avenida Tecnologico No. 1500, Lomas de Santiaguito, 58120 Morelia, MICH, Mexico \\ ${ }^{2}$ Centro de Ciencias Matemáticas, UNAM Campus Morelia, AP 61-3 (Xangari), 58089 Morelia, MICH, Mexico \\ Correspondence should be addressed to Elena Kaikina; ekaikina@matmor.unam.mx
}

Received 3 November 2015; Accepted 18 April 2016

Academic Editor: Pavel Kurasov

Copyright (C) 2016 B. Juarez Campos et al. This is an open access article distributed under the Creative Commons Attribution License, which permits unrestricted use, distribution, and reproduction in any medium, provided the original work is properly cited.

We study the mixed initial-boundary value problem for the capillary wave equation: $i u_{t}+|u|^{2} u=\left|\partial_{x}\right|^{3 / 2} u, t>0, x>0 ; u(x, 0)=$ $u_{0}(x), x>0 ; u(0, t)+\beta u_{x}(0, t)=h(t), t>0$, where $\left|\partial_{x}\right|^{3 / 2} u=(1 / \sqrt{2 \pi}) \int_{0}^{\infty}(\operatorname{sign}(x-y) / \sqrt{|x-y|}) u_{y y}(y) d y$. We prove the global in-time existence of solutions of IBV problem for nonlinear capillary equation with inhomogeneous Robin boundary conditions. Also we are interested in the study of the asymptotic behavior of solutions.

\section{Introduction}

This paper is concerned with the initial-boundary value problem (IBV problem) for the nonlinear capillary wave equation with mixed (Robin) boundary conditions posed on a half-unbounded domain:

$$
\begin{aligned}
i u_{t}+\left|\partial_{x}\right|^{3 / 2} u & =|u|^{2} u, \quad t>0, \quad x>0 ; \\
u(x, 0) & =u_{0}(x), \quad x>0, \\
u(0, t)+\beta u_{x}(0, t) & =h(t), \quad t>0 .
\end{aligned}
$$

Here $\left|\partial_{x}\right|^{3 / 2}$ is a fractional derivative defined by

$$
\left|\partial_{x}\right|^{3 / 2} u=\frac{1}{\sqrt{2 \pi}} \int_{0}^{\infty} \frac{\operatorname{sign}(x-y)}{\sqrt{|x-y|}} u_{y y}(y) d y .
$$

Mixed boundary value problems arise in a variety of applied mathematics, engineering, and physics, such as gas dynamics, nuclear physics, chemical reaction, studies of atomic structures, and atomic calculations. Therefore, the mixed problems have attracted much attention and have been studied by many authors. For detailed description of the mixed boundary conditions, see [1-3] and the references cited therein. This paper is the first attempt to investigate the inhomogeneous mixed initial-boundary value problem for the dispersive fractional nonlinear equation, considering as an example the famous capillary water wave equation (1). Fractional differential equations appear in many applications of the applied sciences, such as the fractional diffusion and wave equations [4], subdiffusion and superdiffusion equations [5], electrical systems [6], viscoelasticity theory [6], control systems [6], bioengineering [7], and finance [8]. Many articles have appeared in the literature, where fractional derivatives are used for a better description of certain material properties. Thus, for example, the fractional NLS model (1) comes from the study of the long-time behavior of solutions to the water waves equations [9]. The operator $i\left|\partial_{x}\right|^{3 / 2}$ corresponds to the dispersive relation of the linearized gravity water wave equations for one-dimensional interfaces with surface tension. Furthermore, thanks to the absence of resonances at the quadratic level, one expects the nonlinear dynamics of water waves to be governed by nonlinearity of cubic type like those appearing in (1). Papers [9-11] addressed some other applications of fractional Schrödinger equations. Works concerning the Cauchy problem for fractional type Schrödinger equations, which address the existence of small solutions, and in particular the question of modified scattering, include [12-14]. In paper [15], it was shown that (1) with dispersive fractional derivative operator of order $1 / 2$ admits global 
solutions whose long-time behavior is not linear. Global existence results and asymptotic behavior of small solutions of Cauchy problem for capillary water wave equation were obtained in [9]. The initial-boundary problems have been much less studied than the Cauchy problems in spite of their importance. The inhomogeneous problems are often called forced ones, when an external force is applied to a system. Frequently the forcing is putted as the inhomogeneous boundary condition. In the case of the initial-boundary value problems there appear new difficulties comparing with the Cauchy problems due to the boundary. For example, in the case of the initial-boundary value problems it is not clear how many of the boundary conditions are required for the well-posedness of the initial-boundary value problem. The answer to this question relies on the construction of the Green function for the linear capillary water wave equation that is interesting on its own. Also it is necessary to take into account the boundary effects which affect the behavior of the solutions. Also usually we ask as less as possible regularity on the initial and boundary data, since the regularity of the solution implies the compatibility conditions on the initial and boundary data. Observe that for the Cauchy problem there is no such complication, in general, and we can ask more regularity on the initial data.

It is well-known that boundary value problems with homogeneous boundary conditions are easier than the corresponding homogeneous problems. However, we present in this paper remarkable results, such as global in-time existence of solutions and its large time asymptotic behavior. In book [16], it was proved that in the case of mixed problem for dissipative equations the solutions obtain more rapid time decay comparing with the case of the Cauchy problem. This phenomenon was also observed for some dispersive equations, such as intermediate-long wave and Benjamin-Ono equations, posed on the positive half-line $[17,18]$. However, there are several important examples of equations whose small solutions do not behave like linear ones, as it is the case for the Schrödinger equation [19]. As we will show below, the same happens for the case of the capillary wave equation (1) with mixed boundary conditions: the cubic nonlinearity is also critical with respect to the large time decay. Theorem 1 below shows that (1) admits global solutions whose longtime behavior is not linear. In particular, a correction of logarithmic type (see (9)) is needed in order to obtain the $t^{-1 / 2}$ decay and the scattering of solutions. Our approach is based on the estimates of the integral equation in the Sobolev spaces. To construct the Green operator on a halfline we adopt the analytic continuation method proposed in the paper [20], based on the Riemann theory. To get smooth solutions we use a method of the decomposition of the critical cubic nonlinearity. A major complication of IBV problem for nonlocal equation (1) on a half-line is that its symbol $K(p)=|p|^{3 / 2}$ is nonanalytic; therefore, we can not apply the Laplace theory directly. The construction of the Green operator involves the solution of the inhomogeneous Riemann problem with discontinuous coefficients. Another difficulty is that the symbol $K(p)$ is dispersive. To get the asymptotics of solution, we need to solve the nonlinear singular integrodifferential equation with Hilbert kernel. We believe that the method developed in this paper could be applied to a wide class of dispersive nonlinear nonlocal equations.

\section{Main Result and Notation}

To state the results of the present paper, we give some notations. The usual direct and inverse Laplace transformations we denote by $\mathscr{L}$ and $\mathscr{L}^{-1}$. The Fourier transform $\mathscr{F}$ and the inverse Fourier transform $\mathscr{F}^{-1}$ are defined as

$$
\begin{aligned}
\mathscr{F} \phi & =\frac{1}{\sqrt{2 \pi}} \int_{\mathbf{R}} e^{-i x \xi} \phi(x) d x, \\
\mathscr{F}^{-1} \phi & =\frac{1}{\sqrt{2 \pi}} \int_{\mathbf{R}} e^{i x \xi} \phi(\xi) d \xi .
\end{aligned}
$$

The usual Fourier sine transform $\mathscr{F}_{s}$ and the Fourier cosine transform $\mathscr{F}_{c}$ are given by

$$
\begin{aligned}
& \mathscr{F}_{s} \phi=\sqrt{\frac{2}{\pi}} \int_{R^{+}} \phi(x) \sin p x d x, \\
& \mathscr{F}_{c} \phi=\sqrt{\frac{2}{\pi}} \int_{R^{+}} \phi(x) \cos p x d x .
\end{aligned}
$$

Define the "distorted" Fourier sine transform $\mathscr{K}_{c}$ and the inverse "distorted" Fourier sine transform $\mathscr{K}_{s}^{*}$ as follows:

$$
\begin{aligned}
& \widehat{\phi}(p)=\mathscr{K}_{s} \phi=\int_{0}^{\infty} \Psi_{s}(x, p) \phi(x) d x, \\
& \phi(x)=\mathscr{K}_{s}^{*} \widehat{\phi}=\frac{1}{2 \pi} \int_{0}^{\infty} \Psi_{s}^{*}(x, p) \widehat{\phi}(p) d p,
\end{aligned}
$$

where

$$
\begin{aligned}
& \Psi_{c}^{*}(x, z) \\
& =e^{i z x} e^{-\Gamma(i)}+e^{-i z x} e^{-\Gamma(-i)}+\frac{1}{2 \pi i} \int_{0}^{-\infty} e^{z q x} \omega(q) d q, \\
& \Psi_{s}=\mathscr{E}^{-}(x, z)-\mathscr{E}^{-}(x,-z), \\
& \mathscr{E}^{-}(x, \pm z)=\lim _{w \rightarrow \pm i, \operatorname{Re} w>0} \frac{1}{2 \pi i} \int_{-i \infty}^{i \infty} \frac{e^{-q z x} e^{\Gamma(q)}}{q-w} d q, \\
& \omega(q)=\frac{3 \sqrt{2}}{2} e^{-\Gamma(q)} \frac{1}{q^{1 / 2}\left((i q)^{3 / 2}-1\right)\left((-i q)^{3 / 2}-1\right)}, \\
& \Gamma(s)=\frac{1}{2 \pi i} \int_{0}^{\infty} \ln (q-s) d \ln \frac{(-i q)^{3 / 2}-1}{(i q)^{3 / 2}-1} .
\end{aligned}
$$

For a detailed study of the properties of $\mathscr{K}_{s} \phi$ and $\mathscr{K}_{s}^{*} \widehat{\phi}$, see below Lemma 3.

Also we introduce the Green operator on a half-line as

$$
\begin{array}{r}
\mathscr{G}(t)=\left(1-\beta \partial_{x}\right) \mathscr{K}_{s}^{*} e^{-i t K(p)} \frac{1}{1+\beta^{2} p^{2}} \mathscr{K}_{s}\left(1+\beta \partial_{x}\right), \\
K(p)=p^{3 / 2}, \operatorname{Re} \beta \neq 0 .
\end{array}
$$


Let $\phi(q)$ be a complex function, defined in $\operatorname{Re} q=0$, which obeys the Hölder condition for all finite $q$ and tends to a definite limit $\phi(\infty)$ as $q \rightarrow \infty$. Then, Cauchy type integral $F(z)=(1 / 2 \pi i) \int_{-i \infty}^{i \infty}(\phi(q) /(q-z)) d q$ constitutes a function which is analytic in the left and right complex semiplanes. Here and below these functions will be denoted by $F^{+}(z)$ and $F^{-}(z)$, respectively. These functions have the limiting values $F^{+}(p)$ and $F^{-}(p)$ at all points of imaginary axis $\operatorname{Re} p=$ 0 , on approaching the contour from the left and from the right, respectively. These limiting values are expressed by Sokhotzki-Plemelj formula (see [20] for these definitions and a discussion of related issues):

$$
F^{ \pm}(p)= \pm \frac{1}{2} \phi(p)+\frac{1}{2 \pi i} \mathrm{VP} \int_{-i \infty}^{i \infty} \frac{\phi(q)}{q-p} d q
$$

The usual Lebesgue space $\mathbf{L}^{p}=\left\{\phi \in \mathbf{S}^{\prime} ;\|\phi\|_{\mathbf{L}^{p}}<\infty\right\}$, where the norm $\|\phi\|_{L^{p}}=\left(\int_{\mathbf{R}^{+}}|\phi(x)|^{p} d x\right)^{1 / p}$ if $1 \leq p<\infty$ and $\|\phi\|_{\mathbf{L}^{\infty}}=\operatorname{esssup}_{x \in \mathbf{R}^{+}}|\phi(x)|$ if $p=\infty$. Weighted Lebesgue space is $\mathbf{L}^{p, k}=\left\{\phi \in \mathbf{S}^{\prime} ;\|\phi\|_{\mathbf{L}^{p, k}}<\infty\right\}$, where the norm $\|\phi\|_{\mathbf{L}^{p, k}}=\left\|\langle\cdot\rangle^{k} \phi(\cdot)\right\|_{\mathbf{L}^{p}}, \quad k \geq 0$.

Weighted Sobolev space is $\mathbf{H}^{m, k}=\left\{\phi \in \mathbf{S}^{\prime}:\|\phi\|_{\mathbf{H}^{m, k}} \equiv\right.$ $\left.\left\|(1+\partial)^{m} \phi\right\|_{\mathbf{L}^{2, k}}<\infty\right\}$, where $m, k \in \mathbf{R}$. The usual Sobolev space is $\mathbf{H}^{m}=\mathbf{H}^{m, 0}$, so the index 0 we omit if it does not cause a confusion. Also $\|\varphi\|_{\mathbf{L}^{\infty}}=\|\varphi\|_{\infty}$, and $\|\varphi\|_{\mathbf{L}^{2}}=\|\varphi\|$.

Let $\mathbf{Y}=\left\{h \in \mathbf{H}^{1,1 / 2+\gamma} ;\|h\|_{\mathbf{Y}}<\infty\right\}$, where $\|h\|_{\mathbf{Y}}=$ $\|h\|_{\mathbf{H}^{1,1 / 2+\gamma}}, \gamma>0$ is small. Different positive constants we denote by the same letter $C$. We denote $\langle t\rangle=1+t,\{t\}=t /\langle t\rangle$.

Our main result is as follows.

Theorem 1. Let $u_{0} \in \mathbf{Z}=\mathbf{H}^{0,1} \cap \mathbf{H}^{1}$, and $h(t) \in \mathbf{Y}$ and the norm $\left\|u_{0}\right\|_{\mathrm{Z}}+\|h\|_{\mathrm{Y}}=\epsilon$. Then, there exists $\epsilon_{0}>0$ such that for all $0<\epsilon<\epsilon_{0}$ the initial-boundary value problem (1) has a unique global solution $u(t) \in \mathbf{C}\left([0, \infty) ; \mathbf{H}^{1}\right)$. Furthermore, there exists a unique final state $\Lambda \in \mathbf{L}^{\infty}$ such that the large time asymptotics

$$
u(t)=\frac{1}{\sqrt{t}} e^{i|\Lambda(s)|^{2} \Lambda(s) \log t} \Lambda(s)+O\left(t^{\gamma-1 / 4-1 / 2}\right)
$$

is true, where $\gamma \in(0,1 / 4)$, and

$$
\begin{gathered}
\Lambda(s)=e^{-\Gamma(i)} \sqrt{\frac{2}{3}} \frac{s^{1 / 4}}{1-i \beta s}\left(\mathscr{K}_{s}\left(1+\beta \partial_{x}\right) u_{0}\right. \\
\left.+2 e^{\Gamma(0)} \int_{0}^{\infty} e^{i|s|^{3 / 2} t} h(t) d t\right)+O\left(\varepsilon^{3}\right) .
\end{gathered}
$$

\section{Sketch of Proof}

Firstly, we construct the Green operator defined by the linear IBV problem posed on a half-line:

$$
\begin{aligned}
i u_{t}+\left|\partial_{x}\right|^{3 / 2} u & =0, \quad t>0, \quad x>0 ; \\
u(x, 0) & =u_{0}(x), \quad x>0, \\
\left(1+\beta \partial_{x}\right) u(0, t) & =h(t), \quad t>0 .
\end{aligned}
$$

To derive an integral representation for the solution of problem (11), we adopt the analytic continuation method proposed in the paper [20]. The Laplace transform of the fractional operator $\left|\partial_{x}\right|^{3 / 2} u$ with respect to space and time variables is

$$
\begin{aligned}
& \mathscr{L}\left|\partial_{x}\right|^{3 / 2} u=\mathbb{P}^{-}\left|\partial_{x}\right|^{3 / 2} u \\
& =\lim _{w \rightarrow p, \operatorname{Re} w>0} \int_{-i \infty}^{i \infty} \frac{1}{q-w}|q|^{3 / 2} \\
& \cdot\left(\widehat{\hat{u}}(q, \xi)-q^{-1} \widehat{u}(0, \xi)-q^{-2} \widehat{u}_{x}(0, \xi)\right) d q,
\end{aligned}
$$

where $\widehat{\widehat{u}}(p, \xi), \widehat{u}(0, \xi)$, and $\widehat{u}_{x}(0, \xi)$ are Laplace transforms of $u(x, t), u(0, t)$, and $u_{x}(0, t)$, respectively.

Thus, since

$$
\begin{aligned}
\widehat{\widehat{u}}(p, \xi) & =\mathbb{P}^{-\widehat{\hat{u}}}(p, \xi) \\
& =\lim _{w \rightarrow p, \operatorname{Re} w>0} \int_{-i \infty}^{i \infty} \frac{1}{q-w} \widehat{\widehat{u}}(q, \xi) d q,
\end{aligned}
$$

applying the Laplace transforms to (11) with respect to space and time variables we obtain

$$
\begin{aligned}
\mathbb{P}^{-} & \{(K(p)+\xi) \widehat{\widehat{u}}(p, \xi) \\
- & {\left.\left[\widehat{u}_{0}(p)+K(p)\left(p^{-1} \widehat{u}(0, \xi)+p^{-2} \widehat{u}_{x}(0, \xi)\right)\right]\right\} } \\
& =0, \quad K(p)=i|p|^{3 / 2} .
\end{aligned}
$$

Let $\Phi^{+}(p, \xi)$ be some complex function analytic in the lefthalf complex plane and $\left|\Phi^{+}(p, \xi)\right|\left\langle C\langle p\rangle^{-\delta}, \delta>0\right.$, such that

$$
\mathbb{P}^{-} \Phi^{+}(p, \xi)=0 \text { for any } \operatorname{Re} \xi>0 \text {. }
$$

Thus, from (14), we obtain

$$
\begin{aligned}
& \widehat{\widehat{u}}(p, \xi)=\frac{1}{K(p)+\xi}\left[\widehat{u}_{0}(p)\right. \\
& \left.\quad+K(p)\left(p^{-1} \widehat{u}(0, \xi)+p^{-2} \widehat{u}_{x}(0, \xi)\right)+\widehat{\Phi}^{+}(p, \xi)\right] .
\end{aligned}
$$

Note that there are three unknown types of data, $\widehat{u}(0, \xi), \widehat{u}_{x}(0$, $\xi)$, and $\widehat{\Phi}^{+}(p, \xi)$ in (16). To find some of these data we use analytic properties of $\widehat{\vec{u}}(p, \xi)$, which is analytic in $\operatorname{Re} p>0$ by the construction. Also, as we see in the left-hand side of (16), $K(p)$ is not analytic. We define the "analyticity switching" functions $Y^{ \pm}(p, \xi)$. Denote $K^{-}(p)=i(i p)^{3 / 2}$. Note that for $\operatorname{Re} p>0$ and $\operatorname{Re} \xi>0$ the equality $K^{-}(p)+\xi=0$ has only one root $k(\xi)=-i(i \xi)^{2 / 3}$ such that $\operatorname{Re} k(\xi)>0$ in $\operatorname{Re} \xi>0$. We make a cut along the negative imaginary axis. Let

$$
\begin{aligned}
& w^{+}=p^{3 / 4}(p-k(\xi))^{-3 / 4}, \\
& w^{-}=p^{3 / 4}(p+k(\xi))^{-3 / 4} .
\end{aligned}
$$

We prove that for $\operatorname{Re} \xi>0$

$$
\text { Ind } \begin{aligned}
\frac{(K(p)+\xi) w^{-}}{\left(K^{-}(p)+\xi\right) w^{+}} & =\frac{1}{2 \pi i} \int_{-i \infty}^{i \infty} d \ln \frac{K(p)+\xi}{K^{-}(p)+\xi} \frac{w^{-}}{w^{+}} \\
& =0 .
\end{aligned}
$$


Therefore, via Index Zero Theorem, we have

$$
\frac{K(p)+\xi}{K^{-}(p)+\xi}=\frac{Y^{+}(p, \xi)}{Y^{-}(p, \xi)},
$$

where

$$
\begin{aligned}
Y^{ \pm} & =e^{\Gamma^{ \pm}(p, \xi)} w^{ \pm} \\
\Gamma(z, \xi) & =\frac{1}{2 \pi i} \int_{-i \infty}^{i \infty} \frac{1}{q-z} \ln \frac{K(q)+\xi}{K^{-}(q)+\xi} \frac{w^{-}}{w^{+}} d q .
\end{aligned}
$$

Via Sokhotski-Plemelj formula, we have

$$
\begin{aligned}
U^{+}-U^{-} & =\frac{\widehat{u}_{0}(p)}{Y^{+}(p, \xi)}, \\
I^{+}-I^{-} & =\frac{K(p) p^{-2}}{Y^{+}(p, \xi)}, \\
J^{+}-J^{-} & =\frac{K(p) p^{-1}}{Y^{+}(p, \xi)},
\end{aligned}
$$

For the analyticity of the function $\widehat{\hat{u}}$ in the right-half complex plane, we need to put the following conditions:

$$
\begin{aligned}
& \frac{1}{Y^{+}} \widehat{\Phi}^{+}(p, \xi)+U^{+}+I^{+} \widehat{u}_{x}(0, \xi)+J^{+} \widehat{u}(0, \xi)=0, \\
& U^{-}(k(\xi), \xi)+J^{-}(k(\xi), \xi) \widehat{u}(0, \xi) \\
& \quad+I^{-}(k(\xi), \xi) \widehat{u}_{x}(0, \xi)=0 .
\end{aligned}
$$

Thus, we must put in the problem (11) only one type of boundary data. Another type of unknown boundary data can be find from relation (24). For example, if we consider Robin boundary condition, $u(0, t)+\beta u_{x}(0, t)=h(t)$, boundary data $u(0, t)$ and $\widehat{u}_{x}(0, \xi)$ are completely determined by

$$
\begin{aligned}
u(0, t) & =h(t)-\beta u_{x}(0, t), \\
\widehat{u}_{x}(0, \xi) & =-\frac{U^{-}(k(\xi), \xi)+\widehat{h}(\xi) J^{-}(k(\xi), \xi)}{\left(I^{-}(k(\xi), \xi)-\beta J^{-}(k(\xi), \xi)\right)},
\end{aligned}
$$

where $\widehat{h}(\xi)$ is Laplace transform of $h(t)$. We will prove that $\widehat{u}_{x}(0, \xi)$ is analytic in $\operatorname{Re} \xi>0$ under condition $\operatorname{Re} \beta \neq$ 0 . Finally substituting (25) and (24) into (23) and applying inverse Laplace transform, we will prove that the solution (11) is represented by $u=\mathscr{G}(t) \widetilde{u}_{0}$, where

$$
\begin{gathered}
\widetilde{u}_{0}=\mathscr{K}_{s} \mathscr{D} u_{0}+2 e^{\Gamma(0)} p^{1 / 2} \int_{0}^{t} e^{i K(p) \tau} h(\tau) d \tau, \\
\mathscr{D}=1+\beta \partial_{x}, \\
\mathscr{G}(t)=\mathscr{D}^{*} \mathscr{K}_{s}^{*} e^{-i t K(p)} \frac{1}{1+\beta^{2} p^{2}}, \quad \mathscr{D}^{*}=1-\beta \partial_{x} .
\end{gathered}
$$

This fact is exploited in Proposition 2 below. Therefore, the IBV problem (1) can be rewritten as

$$
\begin{aligned}
u= & \mathscr{D}^{*} \mathscr{K}_{s}^{*} e^{-i t K(p)} \\
& \cdot \frac{1}{1+\beta^{2} p^{2}}\left(\widetilde{u}_{0}+i \int_{0}^{t} e^{i \tau K(p)} \mathscr{K}_{s} \mathscr{D}|u|^{2} u d \tau\right) .
\end{aligned}
$$

The estimates of the integral equation (27) in the Sobolev spaces yield an a priori estimate of $\|u\|_{\mathbf{H}^{1}}$. To get the $\mathbf{L}^{\infty}$ estimate of $u(x, t)$, we will prove the asymptotics

$$
\begin{aligned}
\mathscr{G}(t) \phi= & e^{-i(\pi / 4)} \frac{e^{(i t / 2) K(\xi)+\Gamma(i)}}{\sqrt{t}} \frac{\xi^{1 / 4}}{1+i \beta \xi} \phi(\xi) \\
& +t^{-1+(1-\gamma) / 4} O\left(\|\hat{\phi}(p)\|_{\mathbf{H}^{1}}\right) .
\end{aligned}
$$

We introduce the new function $\phi$ as

$$
\phi(p, t)=i \mathscr{D}^{*} \mathscr{K}_{s}^{*} e^{-i t K(p)} \int_{0}^{t} e^{i \tau K(p)} \mathscr{K}_{s} \mathscr{D}|u|^{2} u d \tau .
$$

Then, we obtain from (1) and (27)

$$
\phi_{t}=i e^{i K(p) t} \mathscr{K}_{s} u|u|^{2}-2 \frac{p^{1 / 2} e^{i K(p) t}}{1+\beta^{2} p^{2}} h(t) .
$$

In Lemma 7 below, we will prove that the nonlinearity $\mathscr{K}_{s}\left\{u|u|^{2}\right\}$ is decomposed into the resonant term and the remainder such that

$$
\begin{gathered}
\mathscr{K}_{s} u|u|^{2}=-t^{-1} e^{-i t K(p)} \frac{\lambda(1-i \beta p)}{\alpha(p)} \\
\cdot \mathcal{N}\left(\frac{\alpha(p)}{1-i \beta p} \widehat{\phi}(p)\right)+t^{-1-1 / 4+\gamma}(1-i \beta p) \\
\cdot O\left(\left(\|\widehat{\phi}(p)\|_{\mathbf{H}^{1}}+\|h\|_{\mathbf{Y}}+|t u(0, t)|\right)^{3}\right),
\end{gathered}
$$

where $\lambda=e^{-(\Gamma(i)+\Gamma(-i))}>0$ and $\alpha(p)=e^{-\Gamma(i)} \sqrt{2 / 3} p^{1 / 4}$. Also we prove

$$
|t u(0, t)| \leq C\left(\|\widehat{\phi}(p)\|_{\mathbf{H}^{1}}+\|h\|_{\mathbf{Y}}\right)
$$

Then, the estimates of $\|\phi(p)\|_{\infty}$ and $\|\phi(p)\|_{\mathbf{H}^{1}}$ follow. Therefore, along with (28) and (29) we prove the $\mathbf{L}^{\infty}$-estimate of $u(x, t)$. Also we obtain asymptotics of the solution. We construct the Green operator for the initial-boundary value problem in the next section. Section 5 is devoted to several lemmas involved in the proof of the main result. In Section 6, we prove our main result. 


\section{Green Operator}

We consider linearized version of problem (1). We prove the following proposition.

Proposition 2. Let $u_{0}, h \in \mathbf{L}^{1}\left(\mathbb{R}^{+}\right)$, and $\operatorname{Re} \beta \neq 0$. Then, the solution $u(x, t)$ of the initial-boundary value problem (11) has the following integral representation:

$$
\begin{array}{r}
u(x, t)=\mathscr{G} \tilde{u}_{0}=\mathscr{D}^{*} \mathscr{K}_{s}^{*} e^{-i K(p) t} \frac{1}{1+\beta^{2} p^{2}} \widetilde{u}_{0}(p), \\
K(p)=|p|^{3 / 2},
\end{array}
$$

where $\tilde{u}_{0}(p)=\mathscr{K}_{s} \mathscr{D} u_{0}+2 i e^{\Gamma(0)} p^{1 / 2} \int_{0}^{t} e^{i K(p) \tau} h(\tau) d \tau$, and operators $\mathscr{K}_{s}$, and $\mathscr{K}_{s}^{*}$ were given by (6).

Proof. Substituting (25) and (24) into (23) and applying inverse Laplace transform we prove that the solution (11) is represented:

$$
\begin{gathered}
\widehat{\widehat{u}}(p, \xi)=-\frac{Y^{+}(p, \xi)}{K(p)+\xi}\left(U^{-}-\Psi(p, \xi) U^{-}(k(\xi), \xi)\right. \\
\left.-\left(J^{-}(p)-J^{-}(k(\xi)) \Psi(p, \xi)\right) \widehat{h}(\xi)\right)
\end{gathered}
$$

where

$$
\Psi(p, \xi)=\frac{\left(I^{-}(p, \xi)-\beta J^{-}(p, \xi)\right)}{\left(I^{-}(k(\xi), \xi)-\beta J^{-}(k(\xi), \xi)\right)} .
$$

Denote

$$
\mathscr{E}(y, w, \xi)=\frac{1}{2 \pi i} \int_{-i \infty}^{i \infty} \frac{e^{-q y}}{q-w} \frac{1}{Y^{+}(q, \xi)} d q,
$$

where $Y^{+}(q, \xi)$ was introduced by $(20)$. Taking the Laplace transform of (34) with respect to $\xi$ and $p$ variables, we get

$$
u(x, t)=\mathscr{G} u_{0}+\mathscr{H} h,
$$

where

$$
\begin{aligned}
\mathscr{G} u_{0} & =\int_{0}^{\infty} u_{0}(y) G(x, y, t) d y, \\
\mathscr{H} h & =\int_{0}^{t} H(x, t-\tau) h(\tau) d \tau
\end{aligned}
$$

with

$$
\begin{aligned}
& G(x, y, t)=-\left(\frac{1}{2 \pi i}\right)^{2} \int_{-i \infty+\varepsilon}^{i \infty+\varepsilon} d \xi e^{\xi t} \int_{-i \infty}^{i \infty} e^{p x} \\
& \cdot \frac{Y^{+}(p, \xi)}{K(p)+\xi}\left(\mathscr{E}^{-}(y, p, \xi)\right. \\
& \left.-\Psi(p, \xi) \mathscr{E}^{-}(y, k(\xi), \xi)\right) d p, \\
& H(x, t)=\left(\frac{1}{2 \pi i}\right)^{2} \int_{-i \infty+\varepsilon}^{i \infty+\varepsilon} d \xi e^{\xi t} \int_{-i \infty}^{i \infty} e^{p x} \\
& \cdot \frac{Y^{+}(p, \xi)}{K(p)+\xi}\left(J^{-}(p, \xi)\right. \\
& \left.-\Psi(p, \xi) J^{-}(k(\xi), \xi)\right) d p .
\end{aligned}
$$

Now we consider $G(x, y, t)$ given by (39). Using analytic properties of integrand functions for $\operatorname{Im} \xi>0$, we rewrite the function $G$ in the following form:

$$
G(x, y, t)=G_{1}(x, y, t)+G_{2}(x, y, t)
$$

where

$$
\begin{aligned}
& G_{1}(x, y, t)=-\left(\frac{1}{2 \pi i}\right)^{2} \int_{0}^{-\infty e^{-i \varepsilon}} d \xi e^{\xi t} \\
& \cdot \int_{-i \infty}^{i \infty} e^{p x} \frac{Y^{+}(p, \xi)}{K(p)+\xi} \Omega(p, k(\xi)) d p+\left(\frac{1}{2 \pi i}\right)^{2} \\
& \int_{0}^{-\infty e^{i \varepsilon}} d \xi e^{\xi t} \\
& \cdot \int_{-i \infty}^{i \infty} e^{p x} \frac{Y^{+}(p, \xi)}{K(p)+\xi} \Omega\left(p, e^{i(4 \pi / 3)} k(\xi)\right) d p, \\
& G_{2}(x, y, t)=-\left(\frac{1}{2 \pi i}\right)^{2} \int_{0}^{-i \infty e^{-i \varepsilon}} d \xi e^{\xi t} \\
& \cdot \int_{-i \infty}^{i \infty} e^{p x} \frac{Y^{+}(p, \xi)}{K(p)+\xi} \Omega\left(p, e^{i(4 \pi / 3)} k(\xi)\right) d p \\
& -\left(\frac{1}{2 \pi i}\right)^{2} \int_{-i \infty e^{i \varepsilon}}^{0} d \xi e^{\xi t} \\
& \cdot \int_{-i \infty}^{i \infty} e^{p x} \frac{Y^{+}(p, \xi)}{K(p)+\xi} \Omega(p, k(\xi)) d p
\end{aligned}
$$

where

$$
\begin{gathered}
\Omega(p, k(\xi))=\mathscr{E}^{-}(y, p, \xi)-\Psi(p, \xi) \mathscr{E}^{-}(y, k(\xi), \xi) \\
\text { Since } e^{i(4 \pi / 3)} k\left(|\xi| e^{-i \pi}\right)=k\left(|\xi| e^{i \pi}\right) \text {, we get } \\
G_{1}(x, y, t)=0
\end{gathered}
$$

which implies $G(x, y, t)=G_{2}(x, y, t)$. We make the changes of the variables $z=e^{i(4 \pi / 3)} k(\xi)$ and $z=k(\xi)$ in the first and second terms of (43) correspondingly. Using that, by the definitions $K^{-}(k(\xi))=-\xi$ and $K^{-}\left(e^{i(4 \pi / 3)} k(\xi)\right)=-\xi$, we obtain

$$
\begin{aligned}
G(x, y, t)=\left(\frac{1}{2 \pi i}\right)^{2} \int_{-i \infty e^{i \varepsilon}}^{+i \infty e^{-i \varepsilon}} d z e^{-K(z) t} K^{\prime}(z) \int_{-i \infty}^{i \infty} e^{p x} \\
\quad \cdot \frac{Y^{+}(p,-K(z))}{K(p)-K(z)}\left(\mathscr{E}^{-}(y, p,-K(z))\right. \\
\left.-\Psi(p,-K(z)) \mathscr{E}^{-}(y, z,-K(z))\right) d p .
\end{aligned}
$$


Now we consider $J(w,-K(z))$ and $I(w,-K(z))$ given by (22). Via (19) and Cauchy Theorem taking residue in the point $q=$ 0 , we get

$$
\begin{aligned}
& J(w,-K(z))=\frac{1}{2 \pi i} \\
& \cdot \int_{-i \infty}^{i \infty} \frac{1}{q-w} \frac{1}{Y^{+}(q,-K(z))} \frac{K(q)-K(z)+K(z)}{q} \\
& =\frac{1}{2 \pi i} \\
& \cdot \lim _{s \rightarrow 0, \operatorname{Re} s>0} \int_{-i \infty}^{i \infty} \frac{1}{q-w} \frac{1}{Y^{-}(q,-K(z))} \frac{K^{-}(q)-K(z)}{q-s} \\
& +\frac{K(z)}{2 \pi i} \lim _{s \rightarrow 0, \operatorname{Re} s>0} \int_{-i \infty}^{i \infty} \frac{1}{q-w} \frac{1}{Y^{+}(q,-K(z))} \frac{1}{q-s} d q \\
& =-K(z) \frac{1}{w} \frac{1}{Y^{+}(0,-K(z))}-\frac{K(w)-K(z)}{Y^{+}(w,-K(z)) w}
\end{aligned}
$$

and by the same way

$$
I(w,-K(z))=\frac{1}{w} J(w,-K(z))
$$

As a consequence of (47) and (48), from (35) it follows that

$$
\begin{aligned}
& \Psi(p,-K(z)) \\
& =\frac{z^{2}}{p^{2}} \frac{1-\beta p}{1-\beta z}\left(1+\frac{K(p)-K(z)}{Y^{+}(p,-K(z))} \frac{Y^{+}(0,-K(z))}{K(z)}\right) .
\end{aligned}
$$

Substituting (49) into (46), we attain

$$
G(x, y, t)=G_{1}(x, y, t)+G_{2}(x, y, t)
$$

where

$$
\begin{gathered}
G_{1}(x, y, t)=\left(\frac{1}{2 \pi i}\right)^{2} \int_{-i \infty e^{i \varepsilon}}^{+i \infty e^{-i \varepsilon}} d z e^{-K(z) t} K^{\prime}(z) \\
\cdot \lim _{s \rightarrow 0, \operatorname{Re} s<0} \int_{-i \infty}^{i \infty} e^{p x} \frac{Y^{+}(p,-K(z))}{K(p)-K(z)} \\
\cdot\left(\mathscr{E}^{-}(y, p,-K(z))\right. \\
\left.-\frac{z^{2}}{(p-s)^{2}} \frac{1-\beta p}{1-\beta z} \mathscr{E}^{-}(y, z,-K(z))\right) d p, \\
G_{2}(x, y, t)=\left(\frac{1}{2 \pi i}\right)^{2} \int_{-i \infty e^{i \varepsilon}}^{+i \infty e^{-i \varepsilon}} d z e^{-K(z) t} K^{\prime}(z) \\
. \frac{z^{2} Y^{+}(0,-K(z))}{K(z)(1-\beta z)} \mathscr{E}^{-}(y, z,-K(z))(x-\beta) .
\end{gathered}
$$

By Jordan Lemma taking residue in the point $p=-z$, we rewrite $G_{1}$ in the form

$$
\begin{aligned}
G_{1} & (x, y, t)=-G_{2}+\frac{1}{2 \pi i} \int_{-i \infty}^{+i \infty} e^{-K(z) t} Y^{+}(-z,-K(z)) \\
\cdot & e^{-z x}\left(\mathscr{E}^{-}(y,-z,-K(z))\right. \\
& \left.-\frac{1+\beta z}{1-\beta z} \mathscr{E}^{-}(y, z,-K(z))\right)+\left(\frac{1}{2 \pi i}\right)^{2} \\
& \cdot \int_{-i \infty}^{+i \infty} d z e^{-K(z) t} K^{\prime}(z) \int_{0}^{-\infty} e^{p x} \Theta(p,-K(z)) \\
\cdot & \mathscr{E}^{+}(y, p,-K(z)) d p-\left(\frac{1}{2 \pi i}\right)^{2} \\
\cdot & \int_{-i \infty}^{+i \infty} d z e^{-K(z) t} K^{\prime}(z) \int_{0}^{-\infty} e^{p x} \Theta(p,-K(z)) \\
& \cdot \frac{z^{2}}{p^{2}} \frac{1-\beta p}{1-\beta z} \mathscr{E}^{-}(y, z,-K(z)) d p .
\end{aligned}
$$

Here

$$
\begin{aligned}
& \Theta(p,-K(z)) \\
& =\left(\frac{1}{K^{+}(p)-K(z)}-\frac{1}{K^{-}(p)-K(z)}\right), \\
& K^{ \pm}(p)=i(\mp i p)^{3 / 2} .
\end{aligned}
$$

Since integrand is even function with respect to $z$-variable, we get for the last summand of (52)

$$
\begin{aligned}
& \int_{-i \infty}^{+i \infty} d z e^{-K(z) t} K^{\prime}(z) \\
& \quad \cdot \int_{0}^{-\infty} e^{p x} \Theta(p,-K(z)) \mathscr{E}^{+}(y, p) d p=0 .
\end{aligned}
$$

Therefore, $G(x, y, t)=G_{1}(x, y, t)+G_{2}(x, y, t)$ takes the form

$$
\begin{aligned}
& G(x, y, t) \\
& =\left(\frac{1}{2 \pi i}\right)^{2} \int_{0}^{+i \infty} e^{-K(z) t} \mathscr{D}_{x}^{*} \Psi^{*}(x, z) \Psi(y, z), \\
& \mathscr{D}_{x}^{*}=1-\beta \partial_{x},
\end{aligned}
$$


where

$$
\begin{aligned}
\Psi^{*}(x, z)= & Y^{+}(z,-K(z)) e^{z x}-Y^{+}(-z,-K(z)) e^{-z x} \\
& -\frac{K^{\prime}(z)}{2 \pi i} \int_{0}^{-\infty} e^{p x} \Theta(p,-K(z)) \frac{z^{2}}{p^{2}} d p \\
\Psi(y, z)= & \frac{1}{1-\beta z} \mathscr{E}^{-}(y, z,-K(z)) \\
& -\frac{1}{1+\beta z} \mathscr{E}^{-}(y,-z,-K(z)) \\
= & \frac{\Psi_{s}(y, z)+\beta z \Psi_{c}(y, z)}{1-\beta^{2} z^{2}} .
\end{aligned}
$$

Via Lemma $11 Y^{+}(p,-K(z))=e^{-\Gamma\left(p|z|^{-1}\right)}$. Therefore, we make the change of variables $p \rightarrow|z| p, p>0$, to get

$$
\begin{aligned}
\Psi^{*}(x, z)= & e^{-\Gamma(i)} e^{z x}-e^{-\Gamma(-i)} e^{-z x} \\
& -\frac{3}{2} \frac{1}{2 \pi i} \int_{0}^{-\infty} e^{p|z| x} e^{-\Gamma(p)} \Theta(p) d p \\
& \Theta(p)=\frac{1}{p^{2}}\left(\frac{1}{K^{+}(p)-1}-\frac{1}{K^{-}(p)-1}\right) .
\end{aligned}
$$

Also since via Lemma $3 z \Psi_{c}=\partial_{x} \Psi_{s}$,

$$
\Psi(y, z)=\frac{\mathscr{D} \Psi_{s}(y, z)}{1-\beta^{2} z^{2}} .
$$

Thus, finally we obtain

$$
\begin{aligned}
\mathscr{G} \phi & =\int_{0}^{\infty} \phi(y) G(x, y, t) d y \\
& =\mathscr{D}^{*} \mathscr{K}_{s}^{*} e^{-i K(p) t} \frac{1}{1+\beta^{2} p^{2}} \mathscr{K}_{s} \mathscr{D} \phi,
\end{aligned}
$$

$$
K(p)=p^{3 / 2},
$$

where operators $\mathscr{K}_{s}^{*}$ and $\mathscr{K}_{s}$ are defined by (6). By the same way as in the proof of (59), we get

$$
\begin{aligned}
& H(x, t) \\
& \quad=\frac{e^{\Gamma(0)} i}{\pi} \mathscr{D}^{*} \int_{0}^{+\infty} d z e^{-K(z) t} \Psi_{s}^{*}(x, z) \frac{z^{1 / 2}}{1+\beta^{2} z^{2}} d z .
\end{aligned}
$$

$$
\mathscr{H} h=e^{\Gamma(0)} i \mathscr{D}^{*} \mathscr{K}_{s}^{*} e^{-i K(p) t} \frac{p^{1 / 2}}{1+\beta^{2} p^{2}} \int_{0}^{t} e^{i K(p) \tau} h(\tau) d \tau .
$$

Therefore, via (61) and (59), Proposition 2 follows.

\section{Lemmas}

Section 5 is devoted to several lemmas involved in the proof of the main result. Via Proposition 2 by Duhamel principle we have for solution of (1)

$$
\begin{aligned}
u= & \mathscr{D}^{*} \mathscr{K}_{s}^{*} \\
& \cdot \frac{e^{-i K(p) t}}{1+\beta^{2} p^{2}}\left(\widetilde{u}_{0}(p)+i \int_{0}^{t} e^{i K(p) \tau} \mathscr{K}_{s} \mathscr{D N}(u) d \tau\right),
\end{aligned}
$$

where

$$
\begin{aligned}
& \widetilde{\mathcal{u}}_{0}(p)=\mathscr{K}_{s} \mathscr{D} u_{0}+2 i p^{1 / 2} e^{\Gamma(0)} \int_{0}^{t} e^{i K(p) \tau} h(\tau) d \tau, \\
& \mathcal{N}(u)=|u|^{2} u, \\
& K(p)=p^{3 / 2} .
\end{aligned}
$$

Firstly, we prove the main properties of the operators $\mathscr{K}_{s}$, $\mathscr{K}_{c}$ and $\mathscr{K}_{s}^{*}, \mathscr{K}_{c}^{*}$ defined in (6).

Lemma 3. The following estimates are valid:

$$
\begin{aligned}
\left\|\mathscr{K}_{c} \phi\right\|+\left\|\mathscr{K}_{s} \phi\right\| & \leq C\|\phi\|, \\
\left\|\partial_{p} \mathscr{K}_{s} \phi\right\| & \leq C\|\phi\|_{\mathbf{H}^{0,1}}, \\
\left\|\mathscr{D}^{*} \mathscr{K}_{s}^{*} \hat{\phi}\right\|_{\mathbf{H}^{1}} & \leq C\|\hat{\phi}\|_{\mathbf{H}^{0,2}} .
\end{aligned}
$$

Proof. Applying Sokhotski-Plemelj formula, we get

$$
\mathscr{E}^{-}(x,-i z)=-e^{i z x+\Gamma(-i)}
$$

$$
\begin{aligned}
& +\frac{1}{2 \pi i} \int_{-\infty e^{i(\pi / 4)}}^{\infty e^{-i(\pi / 4)}} \frac{e^{-i q z x+\Gamma(i q)}}{q+1} d q \\
= & -e^{i z x+\Gamma(-i)}
\end{aligned}
$$

$$
\begin{gathered}
+\frac{1}{2 \pi i} \int_{-\infty}^{\infty} \frac{e^{-i q z x}\left(e^{\Gamma(i q)}-e^{\Gamma(-i)}\right)}{q+1} d q, \\
\mathscr{E}^{-}(x, i z)=-e^{-i z x+\Gamma(i)}+\frac{1}{2 \pi i} \int_{-\infty e^{i(\pi / 4)}}^{\infty e^{-i(\pi / 4)}} \frac{e^{-q z x+\Gamma(i q)}}{q-1} d q \\
=-e^{-i z x+\Gamma(i)} \\
+\frac{1}{2 \pi i} \int_{-\infty}^{\infty} \frac{e^{-i q z x}\left(e^{\Gamma(i q)}-e^{\Gamma(i)}\right)}{q-1} d q .
\end{gathered}
$$


Thus, for $x>0$,

$$
\begin{aligned}
\Psi_{s}(x, z) & =\mathscr{E}^{-}(x, i z)-\mathscr{E}^{-}(x,-i z) \\
& =e^{i z x+\Gamma(-i)}-e^{-i z x+\Gamma(i)}+\Phi_{s}(x, z), \\
\Phi_{s}(x, z) & =-\frac{1}{\pi} \int_{-\infty e^{i(\pi / 4)}}^{\infty e^{-i(\pi / 4)}} \frac{e^{-q z x+\Gamma(q)}}{q^{2}+1} d q, \\
\Psi_{c}(x, z) & =\mathscr{E}^{-}(x,-z)+\mathscr{E}^{-}(x, z) \\
& =-e^{i z x+\Gamma(-i)}-e^{-i z x+\Gamma(i)}+\Phi_{c}(x, z), \\
\Phi_{c}(x, z) & =\frac{1}{\pi i} \int_{-\infty e^{i(\pi / 4)}}^{\infty e^{-i(\pi / 4)}} \frac{e^{-q z x+\Gamma(q)} q}{q^{2}+1} d q=-\partial_{x} \Phi_{s}(x, z)
\end{aligned}
$$

and as a consequence

$$
\begin{gathered}
i z \Psi_{c}=-\partial_{x} \Psi_{s}, \\
\Psi_{s}(0, z)=0, \\
\partial_{z} \Psi_{s}(x, z)=x \Psi_{c}(x, z), \\
\partial_{z} \Psi_{c}(x, z)=-x \Psi_{s}(x, z) .
\end{gathered}
$$

By the definition,

$$
\begin{aligned}
& \mathscr{K}_{c} \phi=\int_{0}^{\infty} \Psi_{c}(x, z) \phi(x) d x ; \\
& \mathscr{K}_{s} \phi=\int_{0}^{\infty} \Psi_{s}(x, z) \phi(x) d x .
\end{aligned}
$$

From (65) by Plancherel Theorem,

$$
\begin{aligned}
& \left\|\mathscr{K}_{s} \phi\right\| \leq C\left\|\int_{0}^{\infty} e^{i z x} \phi(x) d x\right\| \\
& +C\left\|\int_{0}^{\infty} e^{-i z x} \phi(x) d x\right\| \\
& +C\left\|\int_{-\infty}^{\infty} \frac{\left(e^{\Gamma(i q)}-e^{\Gamma(-i)}\right)}{q+1} d q \int_{0}^{\infty} e^{-i z q x} \phi(x) d x\right\| \\
& +C\left\|\int_{-\infty}^{\infty} \frac{\left(e^{\Gamma(i q)}-e^{\Gamma(i)}\right)}{q-1} d q \int_{0}^{\infty} e^{-i z q x} \phi(x) d x\right\| \\
& \leq C\|\phi\|\left(1+\left\|\int_{-\infty}^{\infty} \frac{\left(e^{\Gamma(i q)}-e^{\Gamma(-i)}\right)}{(q+1) q^{1 / 2}} d q\right\|\right. \\
& \left.+\left\|\int_{-\infty}^{\infty} \frac{\left(e^{\Gamma(i q)}-e^{\Gamma(i)}\right)}{(q-1) q^{1 / 2}} d q\right\|\right) \leq C\|\phi\| .
\end{aligned}
$$

By the same way, we can prove $\left\|\mathscr{K}_{c} \phi\right\| \leq C\|\phi\|$. Since via (68) $i z \Psi_{c}=-\partial_{x} \Psi_{s}$, after integrating by part we have

$$
\begin{aligned}
z \mathscr{K}_{c} \phi & =\int_{0}^{\infty} z \Psi_{c}(x, z) \phi(x) d x \\
& =-\int_{0}^{\infty} \phi(x) \partial_{x} \Psi_{s}(x, z) d x \\
& =\int_{0}^{\infty} \Psi_{s}(x, z) \phi_{x}(x) d x .
\end{aligned}
$$

Therefore,

$$
\left\|z \mathscr{K}_{c} \phi\right\| \leq C\left\|\phi_{x}\right\|
$$

By the definition (6), $\mathscr{D}^{*} \mathscr{K}_{s}^{*} \widehat{\phi}=(1 / 2 \pi) \int_{0}^{\infty} \Psi^{*}(x, z) \widehat{\phi}(z) d z$, where

$$
\begin{aligned}
& \Psi^{*}(x, z)=(1-\beta i z) e^{i z x} e^{-\Gamma(i)}-(1+\beta i z) e^{-i z x} e^{-\Gamma(-i)} \\
& +\widetilde{\Phi}(x, z) \\
& \widetilde{\Phi}(x, z)=C \int_{0}^{-\infty} e^{z q x} e^{-\Gamma(q)} \\
& \cdot \frac{(1-\beta z q)}{q^{1 / 2}\left((i q)^{3 / 2}-1\right)\left((-i q)^{3 / 2}-1\right)} d q .
\end{aligned}
$$

Using analytic properties of integrand function, we can change the contour of integration such that $\operatorname{Re} z q x<0$ for all $q \in \mathscr{C}$. Since $\left|e^{-\Gamma(q)}\right| \leq C$, we have

$$
\begin{aligned}
& \left\|\partial_{x}^{(n)} \int_{0}^{\infty} \widehat{\phi}(p) \widetilde{\Phi}(\cdot, p) d p\right\| \leq C \int_{0}^{\infty}|\widehat{\phi}(p)| d p \\
& \cdot \int_{0}^{\infty} \frac{q^{n}(|p q+i|)^{-1 / 2-\gamma}|1-\beta p q| p^{n}}{q^{1 / 2}\left|(-i q)^{3 / 2}-1\right|\left|(i q)^{3 / 2}-1\right|} d q \\
& \leq C\left\|p^{n}\langle p\rangle^{1 / 2-\gamma} \widehat{\phi}(p)\right\|_{\mathbf{L}^{1}} \leq C\left\|\langle p\rangle^{2} \widehat{\phi}(p)\right\|, \\
& n=0,1 .
\end{aligned}
$$

As a consequence via Plancherel Theorem and estimates (70) and (72), we get

$$
\left\|\mathscr{D}^{*} \mathscr{K}_{s}^{*} \hat{\phi}\right\|_{\mathbf{H}^{1}} \leq C\left\|\langle p\rangle^{2} \hat{\phi}\right\| .
$$

Since by the definition $\partial_{p} \Psi_{s}(x, p)=i x \Psi_{c}(x, p)$ applying $\left\|\mathscr{K}_{c} x \phi\right\| \leq C\|x \phi\|$ we get

$$
\left\|\partial_{p} \mathscr{K}_{s} \phi\right\| \leq C\|\phi\|_{\mathbf{H}^{0,1}}
$$

thus via (70), (72), (75), and (76) the lemma is proved.

We introduce

$$
\begin{aligned}
\xi\left(x t^{-1}\right) & =\xi(\tilde{x})=\left(\frac{2 \tilde{x}}{3}\right)^{2}, \\
K(p) & =p^{3 / 2} .
\end{aligned}
$$


Lemma 4. Let $\widehat{\phi}(p)$ be an analytic in the right-half complex plane, except when $p=x>0$. Then, the asymptotics

$$
\begin{aligned}
\frac{1}{2 \pi} \int_{0}^{+\infty} e^{i p x} e^{-i K(p) t} \widehat{\phi}(p) d p & \\
= & e^{-i(\pi / 4)} \frac{e^{i t(K(\xi) / 2)}}{\sqrt{t K^{\prime \prime}(\xi)}} \widehat{\phi}(\xi) \\
& +t^{-1+(1-\gamma) / 4} O\left(\left\|\hat{\phi}_{p}\right\|+\|\hat{\phi}\|_{\infty}\right), \quad x>0
\end{aligned}
$$

is valid for $x>0$.

Proof. We have, for $\xi>0, \varepsilon=t^{-1 / 2+\gamma}$,

$$
\begin{aligned}
& \frac{1}{2 \pi} \int_{\xi-\varepsilon}^{\xi+\varepsilon} e^{-i\left(K^{\prime \prime}(\xi) / 2\right) t(p-\xi)^{2}} \widehat{\phi}(p) d p \\
& \quad=e^{-i(\pi / 4)} \frac{e^{i t(\sqrt{\xi} / 2)}}{\sqrt{t K^{\prime \prime}(\xi)}} \widehat{\phi}(\xi)+t^{-1 / 4-1 / 2+\gamma}\left\|\phi_{p}\right\| .
\end{aligned}
$$

Indeed, by the direct calculation, it follows that

$$
\begin{aligned}
\int_{\xi-\varepsilon}^{\xi+\varepsilon} & e^{-i \lambda(\xi) t(p-\xi)^{2}} \widehat{\phi}(p) d p \\
= & \widehat{\phi}(\xi) \int_{\xi-\varepsilon}^{\xi+\varepsilon} e^{-i \lambda(\xi) t(p-\xi)^{2}} d p \\
& +\int_{\xi-\varepsilon}^{\xi+\varepsilon} e^{-i \lambda(\xi) t(p-\xi)^{2}}(\widehat{\phi}(p)-\widehat{\phi}(\xi)) d p .
\end{aligned}
$$

Denote $\lambda(\xi)=K^{\prime \prime}(\xi) / 2$. Therefore, changing $t p^{2}=q^{2}$, we get

$$
\begin{aligned}
& \int_{\xi-\varepsilon}^{\xi+\varepsilon} e^{-i \lambda(\xi) t(p-\xi)^{2}} d p=\int_{-\varepsilon}^{\varepsilon} e^{-i \lambda(\xi) t p^{2}} d p \\
& =\frac{1}{\sqrt{t}} \int_{-t^{-\gamma}}^{t^{\gamma}} e^{-i \lambda(\xi) q^{2}} d q=\frac{1}{\sqrt{t \lambda(\xi)}} \int_{-t^{\gamma} \sqrt{\lambda(\xi)}}^{t^{\gamma} \sqrt{\lambda(\xi)}} e^{i q^{2}} d q \\
& =\frac{e^{-i(\pi / 4)}}{\sqrt{t \lambda(\xi)}} \int_{-\infty}^{\infty} e^{-q^{2}} d q=\frac{e^{-i(\pi / 4)} \pi}{\sqrt{t \lambda(\xi)}}, \\
& \int_{-\varepsilon}^{\varepsilon} e^{-i \lambda t p^{2}}(\widehat{\phi}(p+\xi)-\widehat{\phi}(\xi)) d p \\
& =\frac{1}{\sqrt{t}} O\left(\int_{-t^{\gamma}}^{t^{\gamma}} e^{-i \lambda q^{2}}\left(\widehat{\phi}\left(\frac{q}{\sqrt{t}}+\xi\right)-\widehat{\phi}(\xi)\right) d q\right) .
\end{aligned}
$$

Using analytic properties of function $\widehat{\phi}$, we can change the contour of integration such that

$$
\left|e^{-i \lambda q^{2}}\right| \leq C \frac{1}{\left.\left.|1+\lambda| q\right|^{2}\right|^{\gamma}}
$$

and using

$$
\begin{aligned}
\left|\widehat{\phi}\left(\frac{q}{\sqrt{t}}+\xi\right)-\widehat{\phi}(\xi)\right| & =\left|\int_{\xi}^{q / \sqrt{t}+\xi} \widehat{\phi}_{z} d z\right| \\
& \leq C\left\|\widehat{\phi}_{z}\right\| t^{-(1-\gamma) / 4} \sqrt{q}
\end{aligned}
$$

we get

$$
\begin{aligned}
& \left|\int_{-t^{\gamma}}^{t^{\gamma}} e^{-i \lambda q^{2}}\left(\widehat{\phi}\left(\frac{q}{\sqrt{t}}+\xi\right)-\widehat{\phi}(\xi)\right) d q\right| \\
& \leq C\left\|\hat{\phi}_{z}\right\| \int_{0}^{\infty} \sqrt{q} \frac{1}{\left.\left.|1+\lambda| q\right|^{2}\right|^{\gamma}} d q \leq C\left\|\hat{\phi}_{z}\right\|
\end{aligned}
$$

from which it follows (79). We use the stationary phase method. Let $f(p)=-K(p)+p \tilde{x}$.

We define $\xi(\tilde{x})=((2 / 3) \tilde{x})^{2}$ such that $f_{p}(\xi)=-K^{\prime}(\xi)+\tilde{x}=$ 0 . Note that $\left|K^{\prime \prime}(\xi)\right|^{-1 / 2}=(2 / \sqrt{3}) \xi^{1 / 4}$.

Denote $K^{\prime \prime}(\xi)=2 \lambda(\xi)=O\left(\xi^{-1 / 2}\right), \mu(p, \xi)=f(p)-f(\xi)-$ $\lambda(\xi)(p-\xi)^{2}$, and $f(\xi)=K(\xi)-\xi K^{\prime}(\xi)$.

We rewrite $\mathscr{G}_{1} \phi$ as

$$
\begin{aligned}
\mathscr{G}_{1} \phi(x) & =\frac{1}{2 \pi} \int_{0}^{+\infty} e^{i p x} e^{-i K(p) t} \widehat{\phi}(p) d p \\
& =\frac{e^{-\Gamma(i)}}{2 \pi} e^{i t f(\xi)} \int_{\xi-\varepsilon}^{\xi+\varepsilon} e^{i t \lambda(\xi)(p-\xi)^{2}} \hat{\phi}(p) d p+R,
\end{aligned}
$$

where

R

$$
\begin{aligned}
= & \frac{e^{-\Gamma(i)}}{2 \pi} e^{i t f(\xi)} \int_{\xi-\varepsilon}^{\xi+\varepsilon} e^{i t \lambda(\xi)(p-\xi)^{2}}\left(e^{i \mu(p, \xi) t}-1\right) \hat{\phi}(p) d p \\
& +\left(\int_{0}^{\xi-\varepsilon}+\int_{\xi+\varepsilon}^{+\infty}\right) e^{i t f(p, \xi)} \hat{\phi}(p) d p \\
= & R_{1}+R_{2}+R_{3} .
\end{aligned}
$$

Via (79),

$$
\begin{aligned}
e^{i t f(\xi)} \int_{\xi-\varepsilon}^{\xi+\varepsilon} e^{-i t \lambda(p-\xi)^{2}} \widehat{\phi}(p) d p \\
=e^{i(\pi / 4)} \frac{e^{i t(\sqrt{\xi} / 2)}}{\sqrt{t K^{\prime \prime}(\xi)}} \widehat{\phi}(\xi)+t^{-1 / 4-1 / 2+\gamma}\left\|\widehat{\phi}_{p}\right\| .
\end{aligned}
$$

Since $f^{\prime}(\xi)=0$ and $\mu(p, \xi)=O\left(p^{-3 / 2}(p-\xi)^{3}\right)$ after changing the variables $p=p_{1} t^{-1 / 2}+\xi$, we get for $\varepsilon=t^{-1 / 2+\gamma}$

$$
\begin{aligned}
& \left|R_{1}\right| \\
& \leq t^{-2} \int_{-t^{-\gamma}}^{t^{\gamma}} O\left(\left(p t^{-1 / 2}+\xi\right)^{-3 / 2}\right) p^{3} \phi\left(p t^{-1 / 2}+\xi\right) d p \\
& \leq C t^{-2+3 / 4}\|\widehat{\phi}(p)\|_{L^{\infty}} t^{(5 / 2) \gamma} \int_{-t^{-\gamma}}^{t^{\gamma}} p^{3-3 / 2} d p \\
& \leq C t^{-1 / 2-1 / 4}\|\widehat{\phi}(p)\|_{L^{\infty}} . \\
& \text { To estimate } \\
& R_{2}=\int_{\xi+\varepsilon}^{+\infty} e^{i t f(p, \xi)} \widehat{\phi}(p) d p \\
& \quad=\left\{\int_{\xi+\varepsilon}^{2 \xi}+\int_{2 \xi}^{\infty}\right\} e^{i t f(p, \xi)} \hat{\phi}(p) d p=R_{21}+R_{22},
\end{aligned}
$$


we use analytic properties of the function $\widehat{\phi}(p)$. Since $f(p, \xi)=K(p)-p K^{\prime}(\xi) \neq 0$ for $\xi+\varepsilon \leq p \leq 2 \xi$, we can to change the contour of the integration to obtain

$$
\left|e^{i t f(p, \xi)}\right| \leq \frac{C}{|f(p, \xi)| t} \leq C|p|^{-1+\gamma}\langle p\rangle^{-2 \gamma} t^{-1+1 / 4+\gamma}
$$

Therefore,

$$
\gamma \geq 0 \text {. }
$$

$$
R_{21}=\int_{\xi+\varepsilon}^{2 \xi} e^{i t f(p, \xi)} \widehat{\phi}(p) d p=t^{-1+1 / 4+\gamma} O\left(\|\widehat{\phi}(p)\|_{\infty}\right) .
$$

Integrating by part, we get

$$
\begin{aligned}
R_{22} & =\int_{2 \xi}^{\infty} e^{i t f(p, \xi)} \widehat{\phi}(p) d p \\
& =t^{-1}\left(\int_{2 \xi}^{\infty} e^{i t f(p, \xi)} \partial_{p} \frac{\widehat{\phi}(p)}{f_{p}(p, \xi)} d p+\frac{\widehat{\phi}(2 \xi)}{f^{\prime}(2 \xi)}\right),
\end{aligned}
$$

where

$$
\begin{aligned}
\left|\partial_{p} \frac{\hat{\phi}(p)}{f^{\prime}(p)}\right| \leq & \frac{C|\widehat{\phi}(p)|}{\sqrt{p}\left|K^{\prime}(p)-K^{\prime}(\xi)\right|^{2}} \\
& +\frac{C\left|\widehat{\phi}_{p}(p)\right|}{\left|K^{\prime}(p)-K^{\prime}(\xi)\right|} .
\end{aligned}
$$

We have $\left|\widehat{\phi}(2 \xi) / f^{\prime}(2 \xi)\right| \leq C t^{-1+1 / 4}\|\widehat{\phi}(p)\|_{L^{\infty}}$. Also for $2 \xi<$ $p<1$,

$$
\begin{aligned}
& \left|\partial_{p} \frac{\widehat{\phi}(p)}{f^{\prime}(p)}\right| \\
& \quad \leq C t^{1 / 4+\gamma}\left(p^{-1+\gamma}|\widehat{\phi}(p)|+C p^{-1 / 2+\gamma}\left|\widehat{\phi}_{p}(p)\right|\right),
\end{aligned}
$$

and for $p \geq 1$

$$
\begin{aligned}
\left|\partial_{p} \frac{\widehat{\phi}(p)}{f^{\prime}(p)}\right| \leq & \frac{C|\widehat{\phi}(p)|}{\sqrt{p}\left|K^{\prime}(p)-K^{\prime}(\xi)\right|^{2}} \\
& +\frac{C\left|\widehat{\phi}_{p}(p)\right|}{\left|K^{\prime}(p)-K^{\prime}(\xi)\right|} \\
\leq & C p^{-1 / 2}\left(|\widehat{\phi}(p)| p^{-1}+\left|\widehat{\phi}_{p}(p)\right|\right) .
\end{aligned}
$$

Therefore,

$$
\begin{aligned}
\left|R_{22}\right| \leq & t^{-1+1 / 4+\gamma} \int_{2 \xi}^{1}\left(p^{-1}|\widehat{\phi}(p)|+\left|\widehat{\phi}_{p}(p)\right|\right) d p \\
& +t^{-1} \int_{1}^{\infty}\left(p^{-3 / 2}|\widehat{\phi}(p)|+p^{-1 / 2-\gamma} p^{\gamma}\left|\widehat{\phi}_{p}(p)\right|\right) \\
& +C t^{-1+1 / 4}\|\widehat{\phi}(p)\|_{L^{\infty}} \\
\leq & C t^{-1+1 / 4+\gamma}\left(\left\|\widehat{\phi}_{p}(p)\right\|+\|\widehat{\phi}(p)\|_{\infty}\right) .
\end{aligned}
$$

Collecting estimates (91) and (96) from (89), we get

$$
R_{2}=R_{21}+R_{22} \leq C t^{-1+1 / 4+\gamma}\left(\|\widehat{\phi}(p)\|_{\infty}+\left\|\hat{\phi}_{p}(p)\right\|\right) \text {. }
$$

Since $f(p) \neq 0$ for $p \in(0, \xi-\varepsilon)$ using analytic properties of $\widehat{\phi}(p)$, we can the change of the contour of the integration to get

$$
\begin{aligned}
& \left|e^{i t f(p)}\right| \leq \frac{C(t|p|)^{-1}}{\left|(2 / 3) K^{\prime}(p)-K^{\prime}(\xi)\right|} \\
& \quad \leq C t^{-1} p^{-1}\left|\frac{1}{K^{\prime}(\xi)}\right|\left|\frac{1}{-(2 / 3)\left(K^{\prime}(p) / K^{\prime}(\xi)\right)+1}\right| \\
& \quad \leq C t^{-1+1 / 4+\gamma} p^{-1+\gamma} .
\end{aligned}
$$
obtain

Thus, by the same way as in the estimation of $R_{2}$, we

$$
\begin{aligned}
\left|R_{3}\right| & \leq C t^{-1+1 / 4+\gamma}\left\|p^{-1+\gamma} \phi\right\|_{L^{1}(0, \xi)} \\
& \leq C t^{-1+1 / 4+\gamma}\left(\left\|\hat{\phi}_{p}(p)\right\|+\|\widehat{\phi}(p)\|_{\infty}\right) .
\end{aligned}
$$

Finally collecting the estimates (87), (88), (97), and (99) from (85), we obtain

$$
\begin{aligned}
\mathscr{G}_{1} \phi(x)= & e^{-i(\pi / 4)} \frac{e^{i t(K(\xi) / 2)}}{\sqrt{t K^{\prime \prime}(\xi)}} \widehat{\phi}(\xi) \\
& +t^{-1+(1-\gamma) / 4} O\left(\left\|\hat{\phi}_{p}(p)\right\|+\|\widehat{\phi}(p)\|_{\infty}\right), \\
x & >0 .
\end{aligned}
$$

Lemma is proved.

We introduce

$$
\alpha(p)=e^{-\Gamma(i)} \sqrt[2]{\frac{2}{3}} \frac{p^{1 / 4}}{1+i \beta p} .
$$

Lemma 5. The following asymptotics is valid:

$$
\begin{aligned}
\mathscr{D}^{*} & \mathscr{K}_{s}^{*} e^{-i K(p) t} \frac{1}{1+\beta p^{2}} \widehat{\phi} \\
= & e^{-i(\pi / 4)} e^{-\Gamma(i)} \frac{e^{i t(K(\xi) / 2)} \alpha(\xi)}{\sqrt{t}} \widehat{\phi}(\xi) \\
& +t^{-1+(1-\gamma) / 4} O\left(\left\|\hat{\phi}_{p}(p)\right\|+\|\widehat{\phi}(p)\|_{\infty}\right),
\end{aligned}
$$

$$
x>0 .
$$

Proof. By the definition (6), $\mathscr{D}^{*} \mathscr{K}_{s}^{*} \phi=(1 / 2 \pi) \int_{0}^{\infty} \Psi^{*}(x$, $z) \phi(z) d z$, where

$$
\begin{gathered}
\Psi^{*}(x, z)=(1-i \beta z) e^{i z x} e^{-\Gamma(i)}-(1+i \beta z) \\
\cdot e^{-i z x} e^{-\Gamma(-i)}+\widetilde{\Phi}(x, z), \\
\widetilde{\Phi}(x, z)=C \int_{0}^{-\infty} e^{z q x} e^{-\Gamma(q)} \\
\cdot \frac{1-\beta z q}{q^{1 / 2}\left((i q)^{3 / 2}-1\right)\left((-i q)^{3 / 2}-1\right)} d q .
\end{gathered}
$$


Therefore, we rewrite $\mathscr{K}_{s}^{*} e^{-i K(p) t} \widehat{\phi}$ in the form

$$
\mathscr{K}_{s}^{*} e^{-i K(p) t} \hat{\phi}=\mathscr{G}_{1} \phi+\mathscr{G}_{2} \phi+\mathscr{G}_{3} \phi
$$

where

$$
\begin{aligned}
& \mathscr{G}_{1} \phi=\frac{e^{-\Gamma(i)}}{2 \pi} \int_{0}^{\infty} e^{i t(-K(p)+p \tilde{x})} \frac{1}{1+i \beta p} \widehat{\phi}(p) d p, \\
& \mathscr{G}_{2} \phi=\frac{e^{-\Gamma(-i)}}{2 \pi} \int_{0}^{\infty} e^{i t(-K(p)-p \tilde{x})} \frac{1}{1-i \beta p} \widehat{\phi}(p) d p, \\
& \mathscr{G}_{3} \phi=\int_{0}^{\infty} e^{-i t K(p)} \Phi(x, p) \frac{1}{1+\beta^{2} p^{2}} \widehat{\phi}(p) d p .
\end{aligned}
$$

Applying Lemma 4,

$$
\begin{aligned}
\mathscr{G}_{1} \phi= & e^{-i(\pi / 4)} e^{-\Gamma(i)} \frac{e^{i t(K(\xi) / 2)} \alpha(\xi)}{\sqrt{t}} \widehat{\phi}(\xi) \\
& +t^{-1+(1-\gamma) / 4} O\left(\|\widehat{\phi}(p)\|_{\mathbf{H}^{1}}\right) .
\end{aligned}
$$

To obtain asymptotics for $\mathscr{G}_{2} \phi=\left(e^{-\Gamma(-i)} /\right.$ $2 \pi) \int_{0}^{\infty} e^{i t(-K(p)-p \tilde{x})} \widehat{\phi}(p) d p$, we observe that $f_{1}^{\prime}(p)=$ $(-K(p)-p x)^{\prime} \neq 0$ along line of the integration. Therefore, applying standard Laplace method after integrating by part, we can prove

$$
\mathscr{G}_{2} \phi(x)=t^{-1+1 / 4} O\left(\|\widehat{\phi}\|_{\mathbf{H}^{1}}\right)
$$

Also using decay properties of the integrand function, we prove that

$$
\mathscr{G}_{3} \phi=t^{-1+1 / 4} O\left(\|\hat{\phi}\|_{\mathbf{H}^{1}}\right) \text {. }
$$

The lemma is proved.

In the next lemma, we obtain asymptotics of boundary operator $\mathscr{H} h$ given by

$$
\mathscr{H} h=e^{\Gamma(0)} i \mathscr{D}^{*} \mathscr{K}_{s}^{*} \frac{p^{1 / 2}}{1+\beta p^{2}} \int_{0}^{t} e^{-i K(p)(t-\tau)} h(\tau) d \tau .
$$

Lemma 6. The asymptotics

$$
\begin{aligned}
\mathscr{H} h(t)= & e^{-i(\pi / 4)} \frac{e^{i t(K(\xi) / 2)} \alpha(\xi)}{\sqrt{t}} \widehat{h}(0) \\
& +t^{-1+1 / 4+\gamma} O\left(\|h\|_{\mathrm{Y}}\right), \\
\xi & =\frac{4}{9}\left(\frac{x}{t}\right)^{2}, \widehat{h}=\mathscr{L} h
\end{aligned}
$$

are valid, provided the right-hand sides are finite.
Proof. From Lemma 5,

$$
\begin{aligned}
\mathscr{H} h= & \int_{0}^{t} \frac{e^{i(K(\xi(t-\tau)) / 2)(t-\tau)}}{\sqrt{t-\tau}} e^{-i(\pi / 4)} \alpha(\xi(t-\tau)) h(\tau) d \tau \\
& +O\left((t-\tau)^{-3 / 4+\gamma}\right) h(\tau) d \tau \\
= & i t^{-1 / 2} e^{-i(\pi / 4)} \alpha(\xi(t)) e^{i(K(\xi(t)) / 2) t} \int_{0}^{\infty} h(\tau) d \tau \\
& +t^{-1 / 2-1 / 4}\|h\|_{\mathbf{H}_{\infty}^{0,5 / 4}} .
\end{aligned}
$$

Thus, the lemma is proved.

In the next lemma, we prove that $\mathscr{K}_{s}$-transform of the nonlinearity is decomposed into the resonant and remainder terms.

Denote

$$
\begin{aligned}
& \mathcal{N}(u)=i|u|^{2} u \\
& \alpha(p)=e^{-\Gamma(i)} \sqrt[i]{\frac{2}{3}} \frac{p^{1 / 4}}{1+i \beta p} .
\end{aligned}
$$

Lemma 7. Let

$$
u=\mathscr{D}^{*} \mathscr{K}_{s}^{*} e^{-i K(p) t} \frac{\phi(p, t)}{1+\beta^{2} p^{2}} .
$$

Then, the asymptotic formula for large time tholds:

$$
\begin{aligned}
& \mathscr{K}_{s} \mathcal{N}(u)=-\frac{i}{t} e^{-(\Gamma(i)+\Gamma(-i))} e^{-i K(p) t} \\
& \quad \cdot \frac{1}{\alpha(p)}|\alpha(p) \hat{\phi}(p)|^{2} \alpha(p) \hat{\phi}(p) \\
& \quad+C t^{-1-1 / 4}\left(\|\hat{\phi}\|_{\mathbf{H}^{1}}+\|p \widehat{\phi}\|+\langle t\rangle|u(0, t)|+\|h\|_{\mathbf{Y}}\right)^{3} \\
& \quad+C|u(0, t)|^{2+1 / 2} \sqrt{t} .
\end{aligned}
$$

Moreover,

$$
\begin{aligned}
t\left\|e^{i K(p) t} \mathscr{K}_{s} \mathcal{N}(u)\right\|_{\mathbf{H}^{1}} \\
\leq C\|\hat{\phi}\|_{\infty}^{2}\left(\|\hat{\phi}\|_{\mathbf{H}^{1}}+\|p \hat{\phi}\|+\|h\|_{\mathbf{Y}}+|t u(0, t)|\right) \\
\quad+C t^{2}|u(0, t)|^{3} .
\end{aligned}
$$

Proof. We introduce a new function $W(\xi)$ such that

$$
\begin{aligned}
u(x, t) & =\frac{1}{\sqrt{t}} e^{i t f(\xi(\tilde{x}))}\left(W(\xi)+e^{-\xi} \sqrt{t} u(0, t)\right) \\
f(p) & =-K(p)+p K^{\prime}(p), \\
K^{\prime}(\xi) & =\tilde{x} \\
\xi\left(x t^{-1}\right) & =\xi(\tilde{x})=\left(\frac{2 \tilde{x}}{3}\right)^{2} .
\end{aligned}
$$


Note that in view of Lemmas 4 and 6,

$$
\begin{aligned}
W(\xi)= & \phi(\xi) e^{-i(\pi / 4)} \alpha(\xi)+t^{-1 / 4+\gamma}\left(\|\phi\|_{\mathbf{H}^{1}}+\|h\|_{\mathbf{Y}}\right) \\
& -e^{-\xi} \sqrt{t} u(0, t)
\end{aligned}
$$

$W(0)=0$.

Making the change of variables $K^{\prime}(\xi) t=x, \xi \in(0,+\infty)$, we get

$$
\begin{gathered}
\mathscr{K}_{s} \mathcal{N}(u)=i t^{-1 / 2} \int_{0}^{\infty} \Psi_{s}\left(K^{\prime}(\xi) t, p\right) e^{i t f(\xi)} K^{\prime \prime}(\xi) \\
\cdot\left|W+e^{-\xi} \sqrt{t} u(0, t)\right|^{2}\left(W+e^{-\xi} \sqrt{t} u(0, t)\right) d \xi
\end{gathered}
$$

where $f(\xi)=-K(\xi)+\xi K^{\prime}(\xi)$. By the stationary phase method (see proof of Lemma 4), we get

$$
\begin{aligned}
\mathscr{K}_{s} & \left(|u|^{2} u\right)=-\frac{e^{-\Gamma(i)}}{\sqrt{t}} i \int_{0}^{\infty} e^{i t\left(-K(\xi)+(\xi-p) K^{\prime}(\xi)\right)} K^{\prime \prime}(\xi) \\
\cdot & \left|W(\xi)+\sqrt{t} e^{-\xi} u(0, t)\right|^{2} \\
\cdot & \left(W(\xi)+\sqrt{t} e^{-\xi} u(0, t)\right) d \xi+R_{1}=-\frac{i}{t} \\
\cdot & e^{-i K(p) t} e^{-(\Gamma(i)+\Gamma(-i))}|W|^{2} W(p) K^{\prime \prime(1 / 2)}(p)+R_{1},
\end{aligned}
$$

where

$$
\begin{aligned}
R_{1} & =t^{-1-1 / 4} O\left(\left\|p^{-1 / 2}|W(p)|^{2} W(p)\right\|_{\mathbf{H}^{1}}\right. \\
& \left.+\left\|p^{-1 / 2}|W(p)|^{2} \sqrt{t} e^{-\xi} u(0, t)\right\|_{\mathbf{H}^{1}}\right)+|u(0, t)|^{2} \\
& \cdot\left\|p^{-1 / 2}|W(p)| e^{-p}\right\|_{\mathbf{L}^{1}} .
\end{aligned}
$$

Using (120), we obtain

$$
\begin{aligned}
\mathscr{K}_{s}\left(|u|^{2} u\right)= & -\frac{i}{t} e^{-(\Gamma(i)+\Gamma(-i))} e^{-i K(p) t} \\
& \cdot \frac{1}{\alpha(p)}|\alpha(p) \widehat{\phi}(p)|^{2} \alpha(p) \widehat{\phi}(p)+R_{1} \\
& +R_{2},
\end{aligned}
$$

where

$$
\left\|R_{2}\right\|_{\infty} \leq C\left(\frac{1}{\alpha(p)} t^{-1-1 / 4}\left(\|\widehat{\phi}\|_{\mathbf{H}^{1}}+\|h\|_{\mathbf{Y}}\right)\right) .
$$

From (117), we have

$$
\begin{gathered}
W(p)=\sqrt{t} e^{-i t f(p)} \int_{0}^{+\infty} e^{-i t K(z)} \Psi^{*}\left(K^{\prime}(p) t, z\right) \\
\cdot \frac{1}{1+\beta p^{2}} \widehat{\phi}(z) d z-e^{-p} e^{-i t f(p)} u(0, t)
\end{gathered}
$$

where by (6)

$$
\begin{aligned}
\Psi^{*}(x, z)= & (1-i \beta p) e^{i p x} e^{-\Gamma(-i)} \\
& -(1+i \beta p) e^{-i p x} e^{-\Gamma(i)}+\Phi(x, z),
\end{aligned}
$$

with

$$
\begin{aligned}
& \Phi(x, z)=\frac{1}{2 \pi i} C \int_{0}^{-\infty} e^{q z x} e^{-\Gamma(q)} \\
& \cdot \frac{\sqrt{|q|}(1-\beta q z)}{\left((i q)^{3 / 2}-1\right)\left((-i q)^{3 / 2}-1\right)} d q, \\
& \Gamma(s)=\frac{1}{2 \pi i} \int_{0}^{\infty} \ln (q-s) d \ln \frac{(-i q)^{3 / 2}-1}{(i q)^{3 / 2}-1} d q .
\end{aligned}
$$

\section{Denote}

$$
\begin{aligned}
& I(p)=\sqrt{t} e^{-i t f(p)} \int_{0}^{+\infty} e^{-i t K(z)} \Psi^{*}\left(K^{\prime}(p) t, z\right) \\
& \quad \cdot \frac{\widehat{\phi}(z)}{1+\beta z^{2}} d z .
\end{aligned}
$$

We rewrite $I(p)$ in the form

$$
I(p)=I_{1}(p)+I_{2}(p)+I_{3}(p)
$$

where

$$
\begin{aligned}
& I_{1}(p)=\sqrt{t} \int_{0}^{+\infty} e^{i t f(z, p)} \frac{\widehat{\phi}(z)}{1+i \beta z} d z, \\
& \quad f(z, p)=(K(p)-K(z))-(p-z) K^{\prime}(p), \\
& I_{2}(p) \\
& \quad=e^{-\Gamma(-i)} e^{i K(p) t} \sqrt{t} \int_{0}^{\infty} e^{i t\left(-K(z)-z K^{\prime}(p)\right)} \frac{\widehat{\phi}(z)}{1-i \beta z} d z, \\
& I_{3}(p) \\
& \quad=e^{i K(p) t} \sqrt{t} \int_{0}^{\infty} e^{-i t K(z)} \Phi\left(K^{\prime}(p) t, z\right) \frac{\widehat{\phi}(z)}{1+\beta z^{2}} d z .
\end{aligned}
$$

Now we estimate $I_{1}(p)$.

Integrating by the part, we have $I_{1 p}(p)=$ $C t^{1 / 2} \int_{0}^{\infty} e^{i t f(z, p)} \Psi(p, z) d z$, where

$$
\begin{aligned}
& \Psi(p, z) \\
& =\frac{1}{\sqrt{p}}\left(\phi_{1}(z) \frac{C_{1}}{\sqrt{z}}+C_{2}(\sqrt{z}+\sqrt{p}) \phi_{1 z}(z)\right), \\
& \widehat{\phi}_{1}(z)=\frac{\widehat{\phi}(z)}{1+i \beta z} .
\end{aligned}
$$


Advances in Mathematical Physics

13

We have

$$
\begin{aligned}
& \left\|\{p\}^{1 / 4+\gamma}\langle p\rangle^{-1 / 4-\gamma} I_{1 p}\right\|^{2}=t \int_{0}^{\infty}\{p\}^{1 / 2+2 \gamma} \\
& \cdot\langle p\rangle^{-1 / 2-2 \gamma} d p \int_{0}^{\infty} e^{i t f\left(p, z_{1}\right)} \Psi\left(p, z_{1}\right) d z_{1} \\
& \cdot \int_{0}^{\infty} e^{-i t f\left(p, z_{2}\right)} \bar{\Psi}\left(p, z_{2}\right) d z_{2} \\
& =t \int_{0}^{\infty} e^{-i t K\left(z_{1}\right)} d z_{1} \int_{0}^{\infty} e^{i t K\left(z_{2}\right)} d z_{2} \\
& \cdot\left(\int_{0}^{+\infty} d p\{p\}^{1 / 2+2 \gamma}\langle p\rangle^{-1 / 2-2 \gamma} \Psi\left(p, z_{1}\right) \bar{\Psi}\left(p, z_{2}\right)\right. \\
& \left.\cdot e^{i\left(z_{1}-z_{2}\right) K^{\prime}(p) t}\right)=I_{11}+I_{12}+I_{13},
\end{aligned}
$$

where

$$
\begin{aligned}
I_{11} & =t \int_{0}^{\infty} e^{-i t K\left(z_{1}\right)} \frac{\phi_{1}\left(z_{1}\right)}{\sqrt{z_{1}}} d z_{1} \int_{0}^{\infty} e^{i t K\left(z_{2}\right)} \frac{\overline{\phi_{1}}\left(z_{2}\right)}{\sqrt{z_{2}}} d z_{2} \\
& \cdot\left(\int_{0}^{+\infty} d p\{p\}^{1 / 2+2 \gamma}\langle p\rangle^{-1 / 2-2 \gamma} \frac{1}{p} e^{i\left(z_{1}-z_{2}\right) K^{\prime}(p) t}\right) \\
I_{12} & =t \int_{0}^{\infty} e^{-i t K\left(z_{1}\right)} \sqrt{z_{1}} \phi_{1 z}\left(z_{1}\right) d z_{1} \\
& \cdot \int_{0}^{\infty} e^{i t K\left(z_{2}\right)} \sqrt{z_{2}} \bar{\phi}_{1 z}\left(z_{2}\right) d z_{2} \\
\cdot & \left(\int_{0}^{+\infty} d p\{p\}^{1 / 2+2 \gamma}\langle p\rangle^{-1 / 2-2 \gamma} \frac{1}{p} e^{i\left(z_{1}-z_{2}\right) K^{\prime}(p) t}\right) \\
I_{13} & =t \int_{0}^{\infty} e^{-i t K\left(z_{1}\right)} \phi_{1 z}\left(z_{1}\right) d z_{1} \\
& \cdot\left(\int_{0}^{\infty} e^{i t K\left(z_{2}\right)} \bar{\phi}_{1 z}\left(z_{2}\right) d z_{2}\right. \\
& \left(\int_{0}^{+\infty}\{p\}^{1 / 2+2 \gamma}\langle p\rangle^{-1 / 2-2 \gamma} e^{i\left(z_{1}-z_{2}\right) K^{\prime}(p) t}\right) .
\end{aligned}
$$

Since $e^{i\left(z_{1}-z_{2}\right) K^{\prime}(p) t}$ is analytic for all $p \in \mathbb{C}$ except for $p<0$, we can change the contour of the integration such that

$$
\left|e^{i\left(z_{1}-z_{2}\right) K^{\prime}(p) t}\right| \leq C\left(1+\left|z_{1}-z_{2}\right||p|^{1 / 2} t\right)^{-2-\gamma} .
$$

Applying (133) by Young inequality, we have

$$
\begin{aligned}
I_{11} & =t \int_{0}^{\infty} e^{-i t K\left(z_{1}\right)} z_{1}^{-1 / 2} \phi_{1}\left(z_{1}\right) d z_{1} \\
& \cdot \int_{0}^{\infty} e^{i t K\left(z_{2}\right)} z_{2}^{-1 / 2} \phi_{1}\left(z_{2}\right) d z_{2} \\
& \cdot\left(\int_{0}^{+\infty} d p\{p\}^{1 / 2+2 \gamma}\langle p\rangle^{-1 / 2-2 \gamma}\right. \\
& \left.\cdot\left(1+\left|z_{1}-z_{2} \| p\right|^{1 / 2} t\right)^{-2-\gamma}\right) \leq C\left\|z_{1}^{-1 / 2} \phi_{1}\left(z_{1}\right)\right\|^{2} \\
& \cdot \int_{0}^{+\infty} d p\{p\}^{1 / 2+2 \gamma}\langle p\rangle^{-1 / 2-2 \gamma} p^{-3 / 2} d p \\
& \leq C\left\|z_{1}^{-1 / 2} \phi_{1}\left(z_{1}\right)\right\|^{2} .
\end{aligned}
$$

Since $\phi(0)=0$, we have

$$
|\phi(p)| \leq C \sqrt{p}\left\|\phi_{1}\right\|_{\mathbf{H}^{1}}
$$

and as a consequence

$$
\left\|\phi z^{-1 / 2}\right\|^{2} \leq C\|\phi\|_{\mathbf{H}^{1}} .
$$

Thus, from (134),

$$
I_{11} \leq C\|\phi\|_{\mathbf{H}^{1}}^{2} .
$$

Using the same approach, we obtain

$$
I_{12}+I_{13} \leq C\|\phi\|_{\mathbf{H}^{1}}^{2} .
$$

Thus, from (137), (138), and (131), we obtain

$$
\left\|\{p\}^{1 / 4+\gamma}\langle p\rangle^{-1 / 4-\gamma} I_{1 p}\right\| \leq C\left(\left\|\hat{\phi}_{z}\right\|+\|\sqrt{z} \hat{\phi}\|\right) .
$$

By analogy, we can estimate $I_{2 p}$ and $I_{3 p}$ :

$$
\begin{aligned}
& \left\|\{p\}^{1 / 4+\gamma}\langle p\rangle^{-1 / 4-\gamma} I_{2 p}\right\|+\left\|\{p\}^{1 / 4+\gamma}\langle p\rangle^{-1 / 4-\gamma} I_{13}\right\| \\
& \leq C\left(\|\hat{\phi}\|_{\mathbf{H}^{1}}+\|z \widehat{\phi}\|\right),
\end{aligned}
$$

which imply the following estimate for $I_{p}=I_{1 p}+I_{2 p}+I_{3 p}$ :

$$
\left\|\{p\}^{1 / 4+\gamma}\langle p\rangle^{-1 / 4-\gamma} I_{p}\right\| \leq C\left(\|\hat{\phi}\|_{H^{1}}+\|z \hat{\phi}\|\right) .
$$

Note that by the same way we can prove that

$$
\left\|\{p\}^{-1 / 4+\gamma}\langle p\rangle^{-1 / 4-\gamma} I\right\| \leq C\left(\|\hat{\phi}\|_{\mathbf{H}^{1}}+\|z \hat{\phi}\|\right) .
$$

Now we estimate

$$
J(p)=e^{-p} e^{-i t f(p)} u(0, t) .
$$

We have

$$
\begin{aligned}
\left\|\{p\}^{\gamma}\langle p\rangle^{-1 / 4-\gamma} J_{p}\right\| & \leq C t\left|u_{0}(t)\right|, \\
\left\|\{p\}^{-1 / 4+\gamma}\langle p\rangle^{-1 / 4-\gamma} J\right\| & \leq C|u(0, t)| .
\end{aligned}
$$


Thus,

$$
\begin{aligned}
& \left\|\{p\}^{-1 / 4+\gamma}\langle p\rangle^{-1 / 4-\gamma} W\right\| \\
& \leq C\left(\|\widehat{\phi}\|_{\mathbf{H}^{1}}+\|z \widehat{\phi}\|+|u(0, t)|\right), \\
& \left\|\{p\}^{\gamma}\langle p\rangle^{-1 / 4-\gamma} W_{p}\right\| \\
& \leq C\left(\|\hat{\phi}\|_{\mathbf{H}^{1}}+\|z \hat{\phi}\|+\langle t\rangle|u(0, t)|\right) .
\end{aligned}
$$

Since by the construction $W(0, t)=0$, therefore we get

$$
|W(p)| \leq \int_{0}^{p}\left|W_{\xi}\right| d \xi \leq C p^{1 / 2-\gamma}\left\|p^{\gamma} W_{p}\right\| .
$$

Therefore, from (141) and (142), it follows that

$$
\begin{aligned}
\left\|p^{-3 / 2} W^{3}\right\|^{2}= & \int_{0}^{+\infty} p^{-3}|W|^{6} d p \\
= & \int_{0}^{1} p^{-3}|W|^{6} d p \\
& +|W|^{4} \int_{1}^{+\infty}\left|p^{-1 / 4-\gamma} W\right|^{2} d p \\
\leq & C\left\|\{p\}^{\gamma} W_{p}\right\|^{6} \int_{0}^{1} p^{-3} p^{3-6 \gamma} \\
& +C\left\|\langle p\rangle^{-1 / 4-\gamma} W\right\|_{\mathbf{H}^{1}}^{6} .
\end{aligned}
$$

Therefore,

$$
\left\|p^{-1 / 2} W^{3}\right\|_{\mathbf{H}^{1}} \leq C\left(\|\hat{\phi}\|_{\mathbf{H}^{1}}+\|\hat{\phi}\|_{\mathbf{H}^{0,1}}+\langle t\rangle|u(0, t)|\right)^{3} .
$$

Also we can prove

$$
\begin{aligned}
& \left\|p^{-1 / 2}|W(p)| e^{-p}\right\|_{\mathbf{L}^{1}} \\
& \leq C\left(\|\widehat{\phi}\|_{\mathbf{H}^{1}}+\|\widehat{\phi}\|_{\mathbf{H}^{0,1}}+\langle t\rangle|u(0, t)|\right)^{1 / 2}, \\
& \left\|p^{-1 / 2}|W(p)|^{2} \sqrt{t} e^{-p} u(0, t)\right\|_{\mathbf{H}^{1}} \\
& \leq|\sqrt{t} u(0, t)| C\left(\|\widehat{\phi}\|_{\mathbf{H}^{1}}+\|\widehat{\phi}\|_{\mathbf{H}^{0,1}}+\langle t\rangle|u(0, t)|\right)^{2} .
\end{aligned}
$$

Substituting estimates (149) and (150) into (123), we get the first estimate of Lemma 7.
Now we prove the second estimate of Lemma 7. We have

$$
\begin{aligned}
\left\|\partial_{p} e^{i K(p) t} \mathscr{K}_{s} \mathcal{N}(u)\right\|_{\mathbf{L}^{2}(1, \infty)} \\
\leq C\left\|p^{-1} \partial_{p} p e^{i K(p) t} \mathscr{K}_{s} \mathcal{N}(u)\right\|_{\mathbf{L}^{2}(1, \infty)} \\
\quad+C\left\|p^{-1} e^{i K(p) t} \mathscr{K}_{s} \mathcal{N}(u)\right\|_{\mathbf{L}^{2}(1, \infty)}
\end{aligned}
$$

Firstly, we estimate $\left\|p^{-1} \partial_{p} p e^{i K(p) t} \mathscr{K}_{s} \mathscr{N}(u)\right\|_{\mathbf{L}^{2}(1, \infty)}$. Denote $F(p)=p e^{i K(p) t} \mathscr{K}_{s} \mathcal{N}(u)$.

Via (117),

$$
u=t^{-1 / 2} e^{i t f(\xi(\widetilde{x}))}\left(W(\xi)+e^{-\xi} \sqrt{t} u(0, t)\right),
$$

$$
\begin{aligned}
\xi\left(x t^{-1}\right) & =\xi(\tilde{x})=\left(\frac{2 \tilde{x}}{3}\right)^{2}, \\
f(p) & =-K(p)+p K^{\prime}(p)
\end{aligned}
$$

from which it follows that

$$
\begin{aligned}
\partial_{x} u & =e^{i t f(\xi(\widetilde{x}))} t^{-1 / 2}\left(W(\xi)+t^{-1} \sqrt{\xi} W_{\xi}(\xi)\right. \\
& \left.+e^{-\xi} t^{-1 / 2} u(0, t)\right)
\end{aligned}
$$

Applying $\Psi_{s}(0, p)=0$, we get

$$
\begin{aligned}
F(p)= & e^{i K(p) t} \mathscr{K}_{c} \partial_{x}|u|^{2} u \\
& -e^{i K(p) t} \Psi_{c}(0, p)|u(0, t)|^{2} u(0, t),
\end{aligned}
$$

where via (152) and (153)

$$
\begin{aligned}
& \partial_{x}|u|^{2} u=t^{-3 / 2}|W(\xi(\widetilde{x}))|^{2} \\
& \cdot\left(W(\xi)+t^{-1} \sqrt{\xi} W_{\xi}(\xi)+e^{-\xi} t^{-1 / 2} u(0, t)\right) .
\end{aligned}
$$
get

Therefore, making the change of variable $x=K^{\prime}(\xi) t$, we

$$
\begin{aligned}
F(p) & =p e^{i K(p) t} \mathscr{K}_{s} \mathcal{N}(u) \\
& =F_{1}(p)+F_{2}(p)+F_{3}(p),
\end{aligned}
$$

where

$$
\begin{aligned}
& F_{1}(p) \\
& =C t^{-1 / 2} e^{i K(p) t} \int_{0}^{\infty} e^{i t f(z)} \Psi_{c}\left(K^{\prime}(z) t, p\right) \phi_{1}(z) d z, \\
& \phi_{1}(z)=z^{-1 / 2}|W(z)|^{2} W(z),
\end{aligned}
$$




$$
\begin{array}{r}
F_{2}(p) \\
=C t^{-3 / 2} e^{i K(p) t} \int_{0}^{\infty} e^{i t f(z)} \Psi_{c}\left(K^{\prime}(z) t, p\right) \phi_{2}(z) d z, \\
\phi_{2}(z)=|W(z)|^{2} W_{z} .
\end{array}
$$

Now we estimate $\left\|\langle p\rangle^{-1} \partial_{p} F_{1}(p)\right\|$. By the definition (67),

$$
\begin{aligned}
\Psi_{1}(x, z)= & e^{i z x+\Gamma(i)}+e^{-i z x+\Gamma(-i)} \\
& +C \int_{-i \infty \infty e^{i(\pi / 4)}}^{i \infty 0 e^{-i(\pi / 4)}} \frac{e^{-q z x+\Gamma(q)} q}{q^{2}+1} d q, \quad x \geq 0 .
\end{aligned}
$$

We estimate the first "more difficulty" term $e^{i z x+\Gamma(-i)}$ of $\Psi_{c}(x, p)$. Another term can be estimated by the same way using Laplace method (see Lemma 4).
As in the proof of (131) integrating by part, we obtain

$$
F_{1 p}(p)=C t^{-1 / 2} \int_{0}^{\infty} e^{i t f(z, p)} \Phi(p, z) d z,
$$

where $f(z, p)$ and $\Phi(p, z)$ were defined by (124) and (130) with $\phi(z)=z^{-1 / 2}|W(z)|^{2} W(z)$. By the same way as in the proof of estimate (141), we obtain

$$
\begin{aligned}
& t\left\|\langle p\rangle^{-1} F_{1 p}(p)\right\| \\
& \quad \leq C\left\||W(z)|^{3} z^{-1}\right\|+C\left\||W(z)|^{3} z^{-3 / 2}\right\| \\
& +C\left\|z^{-1 / 2}|W(z)| W_{z}\right\| \\
& \quad \leq C\|W\|_{\infty}^{2}\left\|z^{-1 / 4+\gamma}\langle z\rangle^{-2 \gamma} W_{z}\right\|^{2} .
\end{aligned}
$$

By the direct calculation,

$$
\begin{aligned}
F_{2 p}(p)=C t^{-1 / 2} \int_{0}^{\infty} e^{i t f(z, p)} f_{p}(z, p) \phi_{2}(z) d z, \\
\phi_{2}(z)=|W(z)|^{2} W_{z}, f(z, p)=K(p)-K(z)+(z-p) K^{\prime}(p), f_{p}(z, p)=(z-p) K^{\prime \prime}(p) .
\end{aligned}
$$

Therefore, as in the proof of estimate (141), we get

$$
\begin{aligned}
t\left\|p^{-1} F_{2 p}\right\|_{L^{2}(1, \infty)} & \leq C\left\|z^{-1 / 4+\gamma}\langle z\rangle^{-2 \gamma}|W(z)|^{2} W_{z}\right\| \\
& \leq C\|W\|_{\infty}^{2}\left\|z^{-1 / 4+\gamma}\langle z\rangle^{-2 \gamma} W_{z}\right\| .
\end{aligned}
$$

Also we have

$$
\begin{aligned}
& t\left\|p^{-1} F_{3 p}\right\|_{\mathbf{L}^{2}(1, \infty)} \\
& \quad \leq t\left\|p^{-1} \partial_{p} e^{i K(p) t}\right\|_{\mathbf{L}^{2}(1, \infty)}|\mathcal{N}(u(0, t))| \\
& \quad \leq t\left\|p^{-1} \partial_{p} e^{i K(p) t}\right\|_{\mathbf{L}^{2}(1, \infty)}|\mathcal{N}(0, t)| \\
& \quad \leq C t^{2}|u(0, t)|^{3} .
\end{aligned}
$$

Applying (163) and (160) into (156), we obtain

$$
\begin{aligned}
& t\left\|p^{-1} \partial_{p}\left(p e^{i K(p) t} \mathscr{K}_{s} \mathcal{N}(u)\right)\right\|_{\mathbf{L}^{2}(1, \infty)} \\
& \quad \leq C\|W\|_{\infty}^{2}\left\|z^{-1 / 4+\gamma}\langle z\rangle^{-2 \gamma} W_{z}\right\|+C t^{2}|u(0, t)|^{3} .
\end{aligned}
$$

Note that, using the same procedure, we can prove

$$
\begin{aligned}
& t\left\|p^{-1} e^{i K(p) t} \mathscr{K}_{s} \mathcal{N}(u)\right\|_{L^{2}(1, \infty)} \\
& \quad \leq C\|W\|_{\infty}^{2}\left\|z^{-1 / 4+\gamma}\langle z\rangle^{-2 \gamma} W_{z}\right\| .
\end{aligned}
$$

Therefore, via (165) and (166) from (151), we obtain

$$
\begin{aligned}
& t\left\|\partial_{p} e^{i K(p) t} \mathscr{K}_{s} \mathcal{N}(u)\right\|_{L^{2}(1, \infty)} \\
& \quad \leq C\|W\|_{\infty}^{2}\left\|z^{-1 / 4+\gamma}\langle z\rangle^{-2 \gamma} W_{z}\right\|+C t^{2}|u(0, t)|^{3} .
\end{aligned}
$$

For small $|p|<1$ using estimate (130) with $\phi_{1}(z)=$ $|W(z)|^{2} W(z)$, we get

$$
\begin{aligned}
& t\left\|\partial_{p} e^{i K(p) t} \mathscr{K}_{s} \mathcal{N}(u)\right\|_{L^{2}(0,1)} \\
& \quad \leq C\|W\|_{\infty}^{2}\left\|z^{-1 / 4+\gamma}\langle z\rangle^{-2 \gamma} W_{z}\right\| .
\end{aligned}
$$

Thus, applying (141) and (118) along with (168) and (167), we have

$$
\begin{aligned}
& t\left\|\partial_{p} e^{i K(p) t} \mathscr{K}_{s} \mathcal{N}(u)\right\| \\
& \leq C\|\hat{\phi}\|_{\infty}^{2}\left(\|\hat{\phi}\|_{\mathbf{H}^{1}}+\|\hat{\phi}\|_{\mathbf{H}^{0,1}}+\|h\|_{\mathbf{Y}}+|t u(0, t)|\right) \\
& \quad+C t^{2}|u(0, t)|^{3} .
\end{aligned}
$$

By the same way, we can prove

$$
\begin{aligned}
& t\left\|e^{i K(p) t} \mathscr{K}_{s} \mathcal{N}(u)\right\| \\
& \quad \leq C\|\hat{\phi}\|_{\infty}^{2}\left(\|\hat{\phi}\|_{\mathbf{H}^{1}}+\|\hat{\phi}\|_{\mathbf{H}^{0,1}}+\|h\|_{\mathbf{Y}}+|t u(0, t)|\right) .
\end{aligned}
$$

The lemma is proved.

In this lemma, we estimate the Green operator:

$$
\mathscr{G} \widetilde{u}_{0}=\mathscr{D}^{*} \mathscr{K}_{s}^{*} e^{-i K(p) t} \frac{1}{1+\beta^{2} p^{2}} \widetilde{u}_{0},
$$

where

$$
\widetilde{u}_{0}(p)=\mathscr{K}_{s} \mathscr{D} u_{0}+2 i e^{\Gamma(0)} p^{1 / 2} \int_{0}^{t} e^{i K(p) \tau} h(\tau) d \tau .
$$


Lemma 8. The following estimate is valid:

$$
\left\|\mathscr{G} \widetilde{u}_{0}\right\|_{\mathrm{H}^{1}} \leq C\left(\left\|u_{0}\right\|_{\mathbf{H}^{1}}+\|h\|_{\mathbf{H}^{1,1 / 2+\gamma}}\right) .
$$

Proof. Via Lemma 3, $\left\|\mathscr{D}^{*} \mathscr{K}_{s}^{*} \widehat{\phi}\right\|_{\mathbf{H}^{1}} \leq C\left\|\langle p\rangle^{2} \widehat{\phi}\right\|$ and therefore

$$
\left\|\mathscr{G} \widetilde{u}_{0}\right\|_{\mathbf{H}^{1}} \leq C\left\|\tilde{u}_{0}\right\| .
$$

Denote by $\theta(x)$

$$
\theta(x)= \begin{cases}1, & x>0 \\ 0, & x<0 .\end{cases}
$$

Integrating by the part, we get for $|p| \geq 1$

$$
\begin{aligned}
& \int_{0}^{t} e^{i K(p) \tau} h(\tau) d \tau=\frac{1}{i K(p)}\left(h(0)-e^{i K(p) t} h(t)\right. \\
& \left.\quad+\int_{0}^{t} e^{i K(p) \tau} h^{\prime}(\tau) d \tau\right) .
\end{aligned}
$$

Therefore, we rewrite operator $\tilde{u}_{0}$ in the form

$$
\begin{aligned}
\widetilde{u}_{0} & =(1-\theta(p)) \widetilde{u}_{0}+\theta(p) \widetilde{u}_{0} \\
& =\widetilde{u}_{01}+\widetilde{u}_{02},
\end{aligned}
$$

where

$$
\begin{aligned}
\tilde{u}_{01}= & e^{i K(p) t} h(t)(1-\theta(p)) \frac{\sqrt{p}}{i K(p)} \\
& +(1-\theta(p))\left(u_{1}(p)+u_{2}(p)\right) \\
\tilde{u}_{02}= & \theta(p) \tilde{u}_{0},
\end{aligned}
$$

where

$$
\begin{aligned}
& u_{1}(p)=\mathscr{K}_{s} \mathscr{D} u_{0}+\frac{\sqrt{p}}{i K(p)} h(0), \\
& u_{2}(p)=\frac{\sqrt{p}}{i K(p)} \int_{0}^{t} e^{i K(p) \tau} h^{\prime}(\tau) d \tau .
\end{aligned}
$$

Since $\left\|\mathscr{K}_{s} \phi\right\|+\left\|\mathscr{K}_{c} \phi\right\| \leq C\|\phi\|$, we get

$$
\left\|\widetilde{u}_{0}\right\| \leq\left\|\tilde{u}_{01}\right\|+\left\|\tilde{u}_{02}\right\| \leq C\left(\left\|u_{0}\right\|_{\mathbf{H}^{1}}+\|h\|_{\mathbf{H}^{1,1 / 2+\gamma}}\right) \text {. }
$$

Thus, via (174), we get the estimate of the lemma. The lemma is proved.

Now we estimate the nonlinear term of (62).

Lemma 9. The following estimate is valid:

$$
\left\|\int_{0}^{t} \mathscr{G}(t-\tau)|u|^{2} u(\tau) d \tau\right\|_{\mathrm{H}^{1}} \leq C \int_{0}^{t}\|u\|_{\infty}^{2}\|u\|_{\mathrm{H}^{1}} d \tau .
$$

Proof. From Lemma 3, $\|\mathscr{G} \phi\|_{\mathbf{H}^{1}} \leq C\left\|\mathscr{K}_{s} \mathscr{D} \phi\right\|$. Therefore, we have

$$
\begin{aligned}
& \left\|\int_{0}^{t} \mathscr{G}(t-\tau)|u|^{2} u(\tau) d \tau\right\|_{\mathbf{H}^{1}} \\
& \quad \leq C\left\|\mathscr{K}_{s} \int_{0}^{t} e^{i K(p) \tau} \mathscr{D}\left(|u|^{2} u\right) d \tau\right\| .
\end{aligned}
$$

Thus, using $\left\|\partial_{x}|u|^{2} u\right\| \leq C\|u\|_{\infty}^{2}\|u\|_{\mathbf{H}^{1}}$, we get

$$
\left\|\int_{0}^{t} \mathscr{G}(t-\tau)|u|^{2} u(\tau) d \tau\right\|_{\mathbf{H}^{1}} \leq C \int_{0}^{t}\|u\|_{\infty}^{2}\|u\|_{\mathbf{H}^{1}} d \tau .
$$

The lemma is proved.

Lemma 10. Let

$$
\begin{gathered}
u(0, t)=\lim _{x \rightarrow 0} \mathscr{D}^{*} \mathscr{K}_{s}^{*} \frac{e^{-i K(p) t}}{1+\beta^{2} p^{2}}\left(\tilde{u}_{0}(p)\right. \\
\left.+i \int_{0}^{t} e^{i K(p) \tau} \mathscr{K}_{s} \mathscr{D} \mathcal{N}(u) d \tau\right) ;
\end{gathered}
$$

then, the following estimate holds:

$\langle t\rangle|u(0, t)| \leq C\left\|u_{0}\right\|_{\mathrm{Z}}+C\|h\|_{\mathrm{Y}}+C\langle t\rangle$

$$
\cdot\left(\int_{0}^{t / 2} \frac{1}{(t-\tau)}+\int_{t / 2}^{t} \frac{1}{(t-\tau)^{1-\gamma}}\right)\|u\|_{\infty}^{2}(\tau)\|u\|_{\mathbf{H}^{1}}
$$

$\cdot(\tau) d \tau$.

Proof. Taking residue in the point $p=0$, we have

$$
\begin{aligned}
u(0, t)= & \mathscr{K}_{s 0}^{*} e^{-i K(p) t} \widetilde{u}_{0}(p) \\
& +i \mathscr{K}_{s 0}^{*} \int_{0}^{t} e^{-i K(p)(t-\tau)} \mathscr{K}_{s} \mathscr{D} \mathscr{N}(u) d \tau,
\end{aligned}
$$

where

$$
\mathscr{K}_{s 0}^{*} \phi=-\beta e^{\Gamma(0)} \int_{0}^{\infty} \frac{p}{1+\beta p^{2}} \phi(p) d p .
$$

Using the analytic properties of the integrand by Cauchy Theorem, we can change the contour of the integration to get

$$
\begin{aligned}
& \left|\mathscr{K}_{s 0}^{*} e^{-i K(p) t} \widehat{\phi}(p)\right| \\
& \quad \leq C \int_{0}^{\infty} e^{-p^{3 / 2} t}\left|\frac{p}{1+\beta p^{2}}\right||\widehat{\phi}(p)| d p \\
& \quad \leq\|\hat{\phi}\|\left\|e^{-p^{3 / 2} t} \frac{p}{1+\beta p^{2}}\right\| \leq C\langle t\rangle^{-1}\|\widehat{\phi}\| .
\end{aligned}
$$

Therefore,

$$
\begin{aligned}
& \left|\mathscr{K}_{s 0}^{*} \int_{0}^{t} e^{-i K(p)(t-\tau)} \mathscr{K}_{s} \mathscr{D} \mathcal{N}(u) d \tau\right| \\
& \leq C\left|\int_{0}^{t / 2} \frac{1}{(t-\tau)^{n+1}}\left\|\mathscr{K}_{s} \mathscr{D} \mathcal{N}(u)\right\|(\tau) d \tau\right| \\
& \quad+C\left|\int_{t / 2}^{t} \frac{1}{(t-\tau)^{1-\gamma}}\left\|\mathscr{K}_{s} \mathscr{D} \mathcal{N}(u)\right\| d \tau\right| .
\end{aligned}
$$

Since via Lemma 3

$$
\left\|\mathscr{K}_{s} \mathscr{D} \mathcal{N}(u)\right\| \leq C\|\mathscr{D} \mathcal{N}(u)\| \leq C\|u\|_{\infty}^{2}\|u\|_{\mathbf{H}^{1}},
$$


from (189) we get

$$
\begin{aligned}
& \left|\mathscr{K}_{s 0}^{*} \int_{0}^{t} e^{-i K(p)(t-\tau)} \mathscr{K}_{s} \mathscr{D} \mathcal{N}(u) d \tau\right| \\
& \leq C \mid\left(\int_{0}^{t / 2} \frac{1}{(t-\tau)}+\int_{t / 2}^{t} \frac{1}{(t-\tau)^{1-\gamma}}\right)\|u\|_{\infty}^{2}(\tau) \\
& \cdot\|u\|_{\mathbf{H}^{1}}(\tau) d \tau \mid .
\end{aligned}
$$

Also via (180),

$$
\begin{aligned}
\left|\mathscr{K}_{s 0}^{*} e^{-i K(p) t} \widetilde{u}_{0}(p)\right| & \leq C\langle t\rangle^{-1}\left\|\tilde{u}_{0}(p)\right\| \\
& \leq C\langle t\rangle^{-1}\left(\left\|u_{0}\right\|_{\mathbf{H}^{1}}+\|h\|_{\mathbf{H}^{1,1 / 2+\gamma}}\right) .
\end{aligned}
$$

By virtue of (186), (191), and (192) Lemma 10 is proved.

In the next lemma, we consider "analyticity switching" functions $Y^{+}(p,-K(z))=e^{\Gamma^{+}(w, z)} w^{+}$, where

$$
\begin{aligned}
& \Gamma(w, z)=\frac{1}{2 \pi i} \int_{i R} \frac{1}{q-w} \ln \frac{K^{+}(q)-K(z)}{K^{-}(q)-K(z)} \frac{w^{-}}{w^{+}} d q, \\
& K(z)=|z|^{3 / 2}, K^{ \pm}(z)=(\mp i z)^{3 / 2}, w^{ \pm}(q, z)=(q \mp z)^{3 / 4} .
\end{aligned}
$$

Lemma 11. The following formula is valid for $\operatorname{Re} z=0$ :

$$
Y^{+}(p,-K(z))=z^{3 / 4} e^{-\Gamma\left(p|z|^{-1}\right)},
$$

where

$$
\Gamma(s)=\frac{1}{2 \pi i} \int_{0}^{+\infty} \ln (q-s) d \ln \frac{K^{+}(q)-1}{K^{-}(q)-1} .
$$

Moreover,

$$
\left|e^{\Gamma(s)}\right| \leq C\langle|s|\rangle^{-3 / 4}
$$

Proof. Using analytic properties of the integrand function after integrating by part and changing variables $q \rightarrow q|z|$, we get

$$
\begin{aligned}
\Gamma^{+}(p,-K(z)) \\
=-\frac{1}{2 \pi i} \int_{0}^{+\infty} \ln \left(q-p|z|^{-1}\right) d \ln \frac{K^{+}(q)-1}{K^{-}(q)-1} \\
\quad-\frac{3}{4} \mathrm{VP} \frac{1}{2 \pi i} \int_{i R} \ln \left(q-p|z|^{-1}\right)\left(\frac{1}{q-i}-\frac{1}{q+i}\right) .
\end{aligned}
$$

Also taking residue in the point $q=i$, we get

$$
\begin{aligned}
\mathrm{VP} & \frac{1}{2 \pi i} \frac{3}{4} \int_{i R} \ln \left(q-p|z|^{-1}\right)\left(\frac{1}{q-i}-\frac{1}{q+i}\right) \\
& =\ln w^{+} .
\end{aligned}
$$

Thus, substituting (198) into (197), we obtain

$$
\Gamma^{+}(p,-K(z))=-\ln w^{+}+\frac{3}{4} \ln |z|-\Gamma\left(p|z|^{-1}\right)
$$

and as a consequence

$$
Y^{+}(p,-K(z))=z^{3 / 4} e^{-\Gamma\left(p|z|^{-1}\right)} .
$$

Also we have (we make a cut along the negative axis)

$$
\text { ind } \begin{aligned}
\left(\frac{K^{+}(q)-1}{K^{-}(q)-1}\right) & =\frac{1}{2 \pi i} \int_{0}^{\infty} d \ln \frac{K^{+}(q)-1}{K^{-}(q)-1} \\
& =\left.\frac{1}{2 \pi i} \operatorname{Arg} \frac{K^{+}(q)-1}{K^{-}(q)-1}\right|_{0} ^{+\infty}=\frac{3}{4} .
\end{aligned}
$$

Since for $|s|>q>1: \ln (q-s)=\ln |s|+\ln \left(1-q|s|^{-1}\right)=$ $\ln |s|+O\left(q|s|^{-1}\right)$ and $\operatorname{ind}\left(\left(K^{+}(q)-1\right) /\left(K^{-}(q)-1\right)\right)=-3 / 4$ we get

$$
\begin{aligned}
& \Gamma(s)=\frac{1}{2 \pi i} \int_{0}^{+\infty} \ln (q-s) d \ln \frac{K^{+}(q)-1}{K^{-}(q)-1}=\frac{1}{2 \pi i} \\
& \cdot \int_{0}^{|s|} \ln (q-s) d \ln \frac{K^{+}(q)-1}{K^{-}(q)-1}+\frac{1}{2 \pi i} \\
& \cdot \int_{|s|}^{+\infty} \ln (q-s) d \ln \frac{K^{+}(q)-1}{K^{-}(q)-1}=\frac{3}{4} \ln |s| \\
& +\frac{1}{2 \pi i} \int_{0}^{|s|} \ln \left(1-q|s|^{-1}\right)
\end{aligned}
$$$$
\cdot\left(\frac{K^{+^{\prime}}\left(q_{1}\right)}{K^{+}\left(q_{1}\right)-1}-\frac{K^{-^{\prime}}\left(q_{1}\right)}{K^{-}\left(q_{1}\right)-1}\right) d q_{1}+\frac{1}{2 \pi i}
$$$$
\cdot \int_{|s|}^{+\infty} \ln (q-s) d \ln \frac{K^{+}(q)-1}{K^{-}(q)-1}=\frac{3}{4} \ln s
$$$$
+O\left(s^{-\gamma}\right) \text {, }
$$

also for, $|s|<1$,

$\Gamma(s)$

$$
\begin{aligned}
= & \frac{1}{2 \pi i} \int_{|s|}^{+\infty} \ln q\left(\frac{K^{+\prime}(q)}{K^{+}(q)-1}-\frac{K^{-1}(q)}{K^{-}(q)-1}\right) d q \\
& +\frac{1}{2 \pi i} \int_{|s|}^{+\infty} \ln \left(1-\frac{s}{q}\right) \Theta(q) d q+O(1) \\
= & O(1)
\end{aligned}
$$

we get

$$
\left|e^{\Gamma(s)}\right| \leq C
$$

Lemma is proved.

\section{Proof of Theorem 1}

Via Proposition 2 by Duhamel principle, we have for solution of (1)

$$
\begin{aligned}
u= & \mathscr{D}^{*} \mathscr{K}_{s}^{*} \\
& \cdot \frac{e^{-i K(p) t}}{1+\beta^{2} p^{2}}\left(\widetilde{u}_{0}(p)+i \int_{0}^{t} e^{i K(p) \tau} \mathscr{K}_{s} \mathscr{D} \mathcal{N}(u) d \tau\right),
\end{aligned}
$$


where

$$
\begin{aligned}
& \tilde{u}_{0}(p)=\mathscr{K}_{s} \mathscr{D} u_{0}+2 p^{1 / 2} e^{\Gamma(0)} \int_{0}^{t} e^{i K(p) \tau} h(\tau) d \tau \\
& \mathcal{N}(u)=i|u|^{2} u \\
& K(p)=p^{3 / 2}
\end{aligned}
$$

We introduce $\mathbf{X}_{T}=\left\{\phi \in \mathbf{C}\left([0, T] ; \mathbf{H}^{1}\right) ;\|\phi\|_{\mathbf{X}_{T}}<\infty\right\}$, where

$$
\begin{aligned}
& \|f\|_{\mathbf{X}_{T}}=\sup _{t \in[0, T]}\left(\langle t\rangle^{-\gamma}\left\|\left(1+\mathscr{K}_{s}\right) f(t)\right\|_{\mathbf{H}^{1}}\right. \\
& \left.\quad+\langle t\rangle^{1 / 2}\|f(t)\|_{\infty}\right) .
\end{aligned}
$$

The local existence in the function space $\mathbf{X}_{T}$ can be proved by a standard contraction mapping principle. We state it without a proof.

Theorem 12. Let $u_{0} \in \mathbf{Z}=\mathbf{H}^{0,1} \cap \mathbf{H}^{1}, h(t) \in \mathbf{Y}=\mathbf{H}^{1,1 / 2+\gamma} \cap$ $\mathbf{H}^{1}$, and the norm $\left\|u_{0}\right\|_{\mathbf{Z}}+\|h\|_{\mathbf{Y}}=\varepsilon$. Then, there exist $\varepsilon_{0}>0$ and $T>1$ such that for all $0<\varepsilon<\varepsilon_{0}$ the initial value problem (1) has a unique local solution $u \in \mathbf{C}\left([0, T] ; \mathbf{X}_{T}\right)$ with the estimate $\|u\|_{\mathbf{X}_{T}}<\sqrt{\varepsilon}$.

Let us prove that the existence time $T$ can be extended to infinity which then yields the result of Theorem 1. By contradiction, we assume that there exists a minimal time $T>0$ such that the a priori estimate $\|u\|_{\mathbf{X}_{T}}<\sqrt{\varepsilon}$ does not hold; namely, we have $\|u\|_{\mathbf{X}_{T}} \leq \sqrt{\varepsilon}$. get

Applying Lemmas 8 and 9 from (205) and Theorem 12, we

$$
\|u\|_{\mathbf{H}^{1}} \leq C\|u\|_{\mathbf{X}_{T}}^{3} \int_{0}^{t} \frac{1}{\tau^{1-\gamma}} d \tau+C\|h\|_{\mathbf{Y}} \leq C t^{\gamma} \varepsilon^{3 / 2} .
$$

From Lemma 10 and Theorem 12, we obtain

$\langle t\rangle|u(0, t)| \leq C\left\|u_{0}\right\|_{\mathrm{Z}}+C\|h\|_{\mathbf{Y}}+C\langle t\rangle$

$$
\begin{aligned}
& \cdot\left(\int_{0}^{t / 2} \frac{1}{(t-\tau)}+\int_{t / 2}^{t} \frac{1}{(t-\tau)^{1-\gamma}}\right)\|u\|_{\infty}^{2}(\tau)\|u\|_{\mathbf{H}^{1}} \\
& \cdot(\tau) d \tau \leq C\left\|u_{0}\right\|_{\mathbf{Z}}+C\|h\|_{\mathbf{Y}}+C\langle t\rangle\|u\|_{\mathbf{X}_{T}}^{3} \\
& \cdot\left(\int_{0}^{t-1} \frac{1}{(t-\tau)}+\int_{t-1}^{t} \frac{1}{(t-\tau)^{1-\gamma_{1}}}\right) \frac{\tau^{\gamma}}{1+\tau} d \tau \\
& \leq C t^{\gamma} \varepsilon^{3 / 2}
\end{aligned}
$$

where

$$
\begin{aligned}
u(0, t)= & \lim _{x \rightarrow 0} \mathscr{D}^{*} \mathscr{K}_{s}^{*} \frac{e^{-i K(p) t}}{1+\beta^{2} p^{2}} \\
& \cdot\left(\widetilde{u}_{0}(p)+i \int_{0}^{t} e^{i K(p) \tau} \mathscr{K}_{s} \mathscr{D} \mathcal{N}(u) d \tau\right) .
\end{aligned}
$$

To estimate $\mathbf{L}^{\infty}$-norm of the solution, we find the solution in the form

$$
u=\mathscr{D}^{*} \mathscr{K}_{s}^{*} \frac{e^{-i K(p) t}}{1+\beta^{2} p^{2}} \phi(p, t)
$$

Via Proposition 2, we obtain for the new function $\phi(p, t)$ the following ordinary differential equation for $t \geq 1$ depending on a parameter $p \in \mathbf{R}^{+}$:

$$
\begin{aligned}
\mathscr{D}^{*} & \mathscr{K}_{s}^{*} \frac{e^{-i K(p) t}}{1+\beta^{2} p^{2}} \phi_{t}(p, t) \\
& =\mathscr{N}(u)+h(t) \mathscr{D}^{*} \mathscr{K}_{s}^{*} \frac{p^{1 / 2}}{1+\beta^{2} p^{2}},
\end{aligned}
$$

$$
\phi_{1}(p, 1)=\phi^{0}
$$

from which it follows that

$$
\begin{aligned}
\phi_{t}(p, t)= & e^{i K(p) t}\left(1+\beta^{2} p^{2}\right) \mathscr{K}_{s} \mathscr{D}^{*-1} \mathcal{N}(u) \\
& +h(t) p^{1 / 2}, \quad \phi_{1}(p, 1)=\phi^{0} .
\end{aligned}
$$

By the direct calculation,

$$
\begin{aligned}
(1 & \left.+\beta^{2} p^{2}\right) \mathscr{K}_{s} \mathscr{D}^{*-1} \mathcal{N}(u) \\
& =\mathscr{K}_{s} \mathcal{N}(u)+\left.\mathscr{D} \mathscr{N}(u)\right|_{x=0} .
\end{aligned}
$$

Thus, using $\left.|\mathscr{D} \mathcal{N}(u)|_{x=0}|\leq C| u(0, t)\right|^{2}|h(t)|$, we get

$$
\begin{aligned}
\phi_{t}(p, t)= & e^{i K(p) t} \mathscr{K}_{s} \mathcal{N}(u)+h(t) p^{1 / 2} \\
& +\left.\mathscr{D} \mathcal{N}(u)\right|_{x=0}, \quad \phi(p, 1)=\phi^{0} .
\end{aligned}
$$

From Lemma 7, the asymptotic formula for large time $t$ holds:

$$
\begin{aligned}
& \mathscr{K}_{s} \mathcal{N}(u)=-t^{-1} e^{-i t K(p)} \frac{\lambda}{\alpha(p)} \mathcal{N}(\alpha(p) \hat{\phi}(p)) \\
& \quad+t^{-1-1 / 4+\gamma} O\left(\left(\|\widehat{\phi}(p)\|_{\mathbf{H}^{1}}+\|p \widehat{\phi}(p)\|\right.\right. \\
& \left.\quad+|t u(0, t)|)^{3}\right),
\end{aligned}
$$

where $\lambda=e^{-(\Gamma(i)+\Gamma(-i))}>0$ and

$$
\begin{aligned}
& \alpha(p)=e^{-\Gamma(i)} \sqrt{\frac{2}{3}} \frac{p^{1 / 4}}{1+i \beta p} \\
& K(p)=p^{3 / 2} .
\end{aligned}
$$

Firstly, we consider the case of $p \leq t^{1-\gamma}$. Multiplying both sides of (216) by $\alpha(p)$, from (215) we get

$$
\begin{aligned}
& \alpha(p) \phi_{t}(p, t) \\
& =\frac{i \lambda}{t} \mathcal{N}(\alpha(p) \widehat{\phi}(p))+e^{i K(p) t} \alpha(p) p^{1 / 2} h(t) \\
& \quad+\left.\alpha(p) e^{i K(p) t} \mathscr{D} \mathcal{N}(u)\right|_{x=0} \\
& \quad+t^{-1-\gamma} O\left(\left(\|\widehat{\phi}(p)\|_{\mathbf{H}^{1}}+\|p \widehat{\phi}(p)\|+|t u(0, t)|\right)^{3}\right) .
\end{aligned}
$$

Denote

$$
\begin{aligned}
\psi(t) & =\phi_{1} e^{-\lambda i \int_{1}^{t}(1 / \tau)\left|\phi_{1}(p, \tau)\right|^{2} d \tau}, \\
\lambda & =e^{-(\Gamma(i)+\Gamma(-i))} \in \mathbf{R}, \phi_{1}=\alpha(p) \phi,|\psi(t)|=\left|\phi_{1}\right| .
\end{aligned}
$$


Then, from (218) we have

$$
\begin{aligned}
|\psi(t)| & =\left|\phi_{1}\right| \\
\leq & C|\psi(1)|+\int_{0}^{t}|h(\tau)| d \tau \\
& +\int_{0}^{t}|h(\tau)||u(0, \tau)|^{2} d \tau \\
& +O\left(\left(\|\widehat{\phi}(p)\|_{\mathbf{H}^{1}}+\|p \widehat{\phi}(p)\|+|t u(0, t)|\right)^{3}\right) \\
\leq & C|\psi(1)|+\|h\|_{\mathbf{Y}} \\
& +O\left(\left(\|\widehat{\phi}\|_{\mathbf{H}^{1}}+\|\widehat{\phi}\|_{\mathbf{H}^{0,1}}+|t u(0, t)|\right)^{3}\right) .
\end{aligned}
$$

Therefore, from (220) we get

$$
\begin{aligned}
& \|\alpha(p) \phi(p)\|_{\mathbf{L}^{\infty}\left(0, t^{1-\gamma}\right)} \\
& \quad \leq C\left(\|\widehat{\phi}\|_{\mathbf{H}^{1}}+\|\widehat{\phi}\|_{\mathbf{H}^{0,1}}+\|h\|_{\mathbf{Y}}+|t u(0, t)|\right)^{3} .
\end{aligned}
$$

Now we consider the case of $p>t^{1-\gamma}$. We multiply both sides of (215) by $\alpha(p)$ to get

$$
\begin{aligned}
\phi_{t}(p, t)= & C e^{i K(p) t} p^{-1}\left(p \mathscr{K}_{s} \mathcal{N}(u)\right)+h(t) p^{1 / 2} \\
& +\left.\mathscr{D} \mathscr{N}(u)\right|_{x=0}=t^{-1-\gamma} O\left(p \mathscr{K}_{s} \mathcal{N}(u)\right) .
\end{aligned}
$$

Via Lemma 3, we have $p \Psi_{s}(x, p)=\partial_{x} \Psi_{c}(x, p)$. Therefore, integrating by part, we obtain

$$
p \mathscr{K}_{s} \mathcal{N}(u)=\mathscr{K}_{c} \partial_{x} \mathcal{N}(u)-\mathscr{N}(u(0, t)) .
$$

Also since

$$
\begin{aligned}
\left|\mathscr{K}_{c} \partial_{x} \mathcal{N}(u)\right| & \leq C\left\|\partial_{x} \mathcal{N}(u)\right\|_{\mathbf{L}^{1}} \leq C\|u\|_{\infty}^{2}\|u\|_{\mathbf{H}^{1}} \\
& \leq C t^{-1+\gamma}\|u\|_{\mathbf{X}_{T}}^{3},
\end{aligned}
$$

from (222) we obtain $\alpha(p) \phi_{t}(p, t)=t^{-1-\gamma} \varepsilon^{3 / 2}$ and as a consequence

$$
\|\phi(p)\|_{\mathbf{L}^{\infty}\left(t^{1-\gamma}, \infty\right)} \leq C\|u\|_{\mathbf{X}_{T}}^{3} .
$$

Thus, along with (221), applying $|t u(0, t)| \leq C\|u\|_{\mathbf{X}_{T}}$ we get

$$
\|\alpha(p) \phi(p)\|_{\mathbf{L}^{\infty}} \leq C\left(\|\phi\|_{\mathbf{H}^{1}}+\|h\|_{\mathbf{Y}}+\|u\|_{\mathbf{X}_{T}}\right)^{3} .
$$

Via Lemmas 4 and 6, we have

$$
\begin{aligned}
u(x, t)= & \mathscr{K}_{s}^{*} e^{-i K(p) t} \widehat{\phi}(p, t) \\
= & -\frac{\lambda}{\sqrt{t}} e^{i(\sqrt{\xi} / 2) t} \alpha(\xi) \phi(\xi) \\
& +t^{-1 / 2-1 / 4}\left(\|\phi\|_{\mathbf{H}^{1}}+\|h\|_{\mathbf{Y}}\right)^{3} .
\end{aligned}
$$

Thus, from estimate (226) along with Theorem 12 it follows that

$$
\begin{aligned}
\sup _{t \geq 0}\langle t\rangle^{1 / 2}\|u(t)\|_{\infty} \\
\leq C \sup _{t \geq 0}\left\|p^{1 / 4} \phi\right\|_{\infty} \\
\quad+C\langle t\rangle^{-1 / 4}\left(\|\phi\|_{\mathbf{H}^{1}}+\|p \phi\|+\|h\|_{\mathbf{Y}}+\|u\|_{\mathbf{X}_{T}}\right)^{3} .
\end{aligned}
$$

Now we estimate $\|\phi\|_{\mathbf{H}^{1}}$. Via (212),

$$
\begin{aligned}
\|\phi(p, t)\|_{\mathbf{H}^{1}} \leq & C \int_{0}^{t}\left\|e^{i K(p) \tau} \mathscr{K}_{s} \mathcal{N}(u)\right\|_{\mathbf{H}^{1}} d \tau \\
& +\|\phi(0)\|_{\mathbf{H}^{1}}, \\
\|p \phi(p, t)\| \leq & C \int_{0}^{t}\left\|e^{i K(p) \tau} p \mathscr{K}_{s} \mathcal{N}(u)\right\| d \tau \\
& +\|p \phi(0)\| .
\end{aligned}
$$

From estimate (116) of Lemma 7, we have

$$
\begin{aligned}
& t\left\|e^{i t K(p)} \mathscr{K}_{s} \mathcal{N}(u)\right\|_{\mathbf{H}^{1}} \\
& \quad=O\left(\|\phi\|_{\mathbf{L}^{\infty}}^{2}\left(\|\phi\|_{\mathbf{H}^{1}}+\|p \phi\|+|t u(0, t)|\right)\right) .
\end{aligned}
$$

From Lemma 3 we have $\|\phi\|_{\mathbf{H}^{1,1}} \leq C\left\|p\left(1+\partial_{p}\right) \mathscr{K}_{s} \phi\right\|$ and therefore

$$
\|\phi(0)\|_{\mathbf{H}^{1}} \leq\left\|\langle x\rangle u_{0}(x)\right\|+\left\|u_{0 x}(x)\right\| \leq C\left\|u_{0}\right\|_{\mathbf{Z}} .
$$

Applying (230) and (231) into (229), we get

$$
\begin{aligned}
\|\phi\|_{\mathbf{H}^{1}} & \\
\leq & C\left\|u_{0}\right\|_{\mathbf{Z}}+C\|h\|_{\mathbf{Y}} \\
& +C\|\hat{\phi}\|_{\infty}^{2} \int_{0}^{t} \frac{1}{\tau^{1-\gamma}}\left(\|\phi\|_{\mathbf{H}^{1}}+\|p \phi\|+\|u\|_{\mathbf{X}_{T}}\right) d \tau .
\end{aligned}
$$

Also, we have

$$
\begin{aligned}
\|p \phi(p, t)\| \leq & C\left\|u_{0}\right\|_{\mathbf{Z}}+C\|h\|_{\mathbf{Y}} \\
& +C\left\|\int_{0}^{t} e^{i K(p) \tau} p \mathscr{K}_{s} \mathcal{N}(u) d \tau\right\| d \tau .
\end{aligned}
$$

We have

$$
p \mathscr{K}_{s} \mathcal{N}(u)=\mathscr{K}_{s} \partial_{x} \mathcal{N}(u)+u(0, t)|u(0, t)|^{2} .
$$

Integrating by part, we attain for $|p|>1$

$$
\begin{aligned}
& \left.\left|\int_{0}^{t} e^{i K(p) \tau} u(0, \tau)\right| u(0, \tau)\right|^{2} d \tau \mid \leq C p^{-3 / 2}\left(|u(0,0)|^{3}\right. \\
& \left.\quad+|u(0, t)|^{3}+\int_{0}^{t}\left|u_{\tau}(0, \tau)\right||u(0, \tau)|^{2} d \tau\right) \\
& \quad=O\left(p^{-3 / 2}\right) .
\end{aligned}
$$


From estimates (221) and (232) along with Theorem 12, it follows that

$$
\|\phi\|_{\mathbf{H}^{1}} \leq C t^{\gamma} \varepsilon^{3 / 2} .
$$

Via (208), (226), and (236), we get that

$$
\|u\|_{\mathbf{X}_{T}} \leq C \varepsilon<\sqrt{\varepsilon}
$$

which implies the desired contradiction. Thus, there exists a unique global solution $u \in \mathbf{C}([0, \infty) ; \mathbf{X})$ of (27) with the time decay estimate $\sup _{t \geq 1}\langle t\rangle^{1 / 2}\|u(t)\|_{\infty} \leq C$.

We now prove the asymptotics of solutions. Denote

$$
\begin{aligned}
\psi(t)=\alpha(p) \phi e^{-\lambda i|\alpha(p) \phi(p, t)|^{2} \log t} e^{-\lambda i \Phi(t)}, & \\
\lambda & =e^{-(\Gamma(i)+\Gamma(-i))}>0,
\end{aligned}
$$

where

$$
\begin{aligned}
& \phi(p, t)=\frac{1}{1+\beta p^{2}}\left(\mathscr{K}_{s} \mathscr{D} u_{0}\right. \\
& \left.-e^{\Gamma(0)} p^{1 / 2} \int_{0}^{\infty} e^{i K(p) \tau} h(\tau) d \tau+\mathscr{K}_{s} \mathscr{D} \mathcal{N}(u)\right) .
\end{aligned}
$$

By (215), we have

$$
\|\psi(t)-\psi(s)\|_{\infty} \leq C \varepsilon^{3 / 2} \int_{s}^{t} \tau^{\gamma-5 / 4} d \tau \leq C \varepsilon^{3 / 2} s^{\gamma-1 / 4}
$$

with $\gamma \in(0,1 / 4)$ and

$$
\Phi(t)=\int_{1}^{t}\left(|\psi(p, \tau)|^{2}-|\psi(p, t)|^{2}\right) \frac{d \tau}{\tau} .
$$

Thus, we see that there exists a unique final state $\psi_{+} \in \mathbf{L}^{\infty}$ such that $\left\|\psi(p, t)-\psi_{+}(p)\right\|_{\infty} \leq C \varepsilon^{3 / 2} t^{\gamma-1 / 4}$. We consider the asymptotics of the phase function $\Phi(t)$ given by (241). By a direct calculation, we have

$$
\begin{aligned}
& \Phi(p, t)-\Phi(p, s) \\
& \quad=\left(\int_{s}^{t}\left(|\psi(p, \tau)|^{2}-|\psi(p, t)|^{2}\right) \frac{d \tau}{\tau}\right. \\
& \left.\quad+\left(|\psi(p, t)|^{2}-|\psi(p, s)|^{2}\right) \log s\right),
\end{aligned}
$$

where $1<s<\tau<t$. Hence,

$$
\begin{aligned}
& \|\Phi(p, t)-\Phi(p, s)\|_{\infty} \\
& \leq C \int_{s}^{t}\|\psi(\tau)-\psi(t)\|_{\infty}\left(\|\psi(\tau)\|_{\infty}+\|\psi(t)\|_{\infty}\right) \frac{d \tau}{\tau} \\
& \quad+C\|\psi(s)-\psi(t)\|_{\infty}\left(\|\psi(s)\|_{\infty}+\|\psi(t)\|_{\infty}\right) \log s \\
& \leq C \varepsilon^{5 / 2} \int_{s}^{t} \tau^{\gamma-5 / 4} d \tau+C \varepsilon^{5 / 2} s^{\gamma-5 / 4} \log s
\end{aligned}
$$

from which it follows that there exists a unique real valued function $\Phi_{+}(p)$ such that

$$
\left\|\Phi(t)-\Phi_{+}\right\|_{\infty} \leq C \varepsilon^{3 / 2} t^{\gamma-1 / 4} .
$$

Therefore, we have the asymptotics of the phase function

$$
\begin{aligned}
\int_{1}^{t}|\psi(p, \tau)|^{2} \frac{d \tau}{\tau}= & \Phi_{+}+\left(\Phi(t)-\Phi_{+}\right)+\widetilde{\psi}_{+} \log t \\
& +\left(\widetilde{\psi}(t)-\widetilde{\psi}_{+}\right) \log t \\
= & \Phi_{+}(p) \\
& +\left(K^{\prime \prime}(p)\right)^{-1 / 2}\left|\psi_{+}(p)\right|^{2} \log t \\
& +O\left(t^{-\gamma}\right),
\end{aligned}
$$

where $\widetilde{\psi}=|\psi(p, \tau)|^{2}$. We also have

$$
\begin{aligned}
\alpha(p) & \phi(p)=e^{\lambda i \int_{1}^{t}|\psi(p)|^{2}(d \tau / \tau)} \psi(p, t) \\
= & e^{i \Phi_{+}-\lambda i \Psi(p) \log t} \psi_{+}(p) \\
& +e^{i \Phi_{+}-3 i\langle p\rangle^{-1}\left|\psi_{+}\right|^{2} \log t}\left(\psi(p, t)-\psi_{+}\right) \\
& +\psi(p, t)\left(e^{\lambda i \int_{1}^{t}|\psi(p)|^{2}(d \tau / \tau)}-e^{i \Phi_{+}-\lambda i \Psi(p) \log t}\right) .
\end{aligned}
$$

Collecting these estimates, we find

$$
\begin{aligned}
\| \alpha & (p) \widehat{\phi}_{1}(p, t)-e^{i \Phi_{+}-\lambda i \Psi(p) \log t} \psi_{+}(p) \|_{\infty} \\
& \leq C\left\|\psi(t)-\psi_{+}\right\|_{\infty}+\left\|\psi_{+}\right\|_{\infty}\left\|\Phi(t)-\Phi_{+}\right\|_{\infty} \\
& +\left\|\psi_{+}\right\|_{L^{\infty}}\left\|\psi(t)-\psi_{+}\right\|_{\infty}\left(\left\|\psi_{+}\right\|_{\infty}+\|\psi(t)\|_{\infty}\right) \log t \\
& \leq C \varepsilon^{3 / 2} t^{\gamma-1 / 4} .
\end{aligned}
$$

Via Lemma 4, estimate (247) means that

$$
\left\|u(t)-\frac{e^{i \log t}}{\sqrt{t}} \Lambda\left(\frac{x}{t}\right)\right\|_{\infty} \leq C \varepsilon^{3 / 2} t^{\gamma-1 / 4-1 / 2},
$$

where $\Lambda(s)=e^{-i(\pi / 4)} \psi_{+}(s) \exp i \Phi_{+}(s)$. Theorem 1 is now proved.

\section{Competing Interests}

The authors declare that they have no competing interests.

\section{Acknowledgments}

This work is partially supported by CONACYT and PAPIIT IN100114.

\section{References}

[1] R. P. Agarwal, D. O’Regan, and S. Staněk, "Positive and maximal positive solutions of singular mixed boundary value problem," Central European Journal of Mathematics, vol. 7, no. 4, pp. 694716, 2009.

[2] T. Buchukuri, O. Chkadua, and D. Natroshvili, "Mixed boundary value problems of thermopiezoelectricity for solids with interior cracks," Integral Equations and Operator Theory, vol. 64, no. 4, pp. 495-537, 2009. 
[3] R. Brown, I. Mitrea, M. Mitrea, and M. Wright, "Mixed boundary value problems for the stokes system," Transactions of the American Mathematical Society, vol. 362, no. 3, pp. 1211$1230,2010$.

[4] W. Wyss, "The fractional diffusion equation," Journal of Mathematical Physics, vol. 27, no. 11, pp. 2782-2785, 1986.

[5] R. Metzler and J. Klafter, "The random walk's guide to anomalous diffusion: a fractional dynamics approach," Physics Reports, vol. 339, no. 1, 77 pages, 2000.

[6] J. Sabatier, O. P. Agrawal, and J. A. T. Machado, Advances in Fractional Calculus: Theoretical Developments and Applications in Physics and Engineering, Springer, Amsterdam, The Netherlands, 2007.

[7] R. L. Magin, Fractional Calculus in Bioengineering, Begell House Publisher, Danbury, Conn, USA, 2006.

[8] E. Scalas, R. Gorenflo, and F. Mainardi, "Fractional calculus and continuous-time finance," Physica A: Statistical Mechanics and Its Applications, vol. 284, no. 1-4, pp. 376-384, 2000.

[9] P. Germain, N. Masmoudi, and J. Shatah, "Global existence for capillary water waves," Communications on Pure and Applied Mathematics, vol. 68, no. 4, pp. 625-687, 2015.

[10] X. Guo and M. Xu, "Some physical applications of fractional Schrödinger equation," Journal of Mathematical Physics, vol. 47, no. 8, Article ID 082104, 9 pages, 2006.

[11] N. Laskin, "Fractional Schrödinger equation," Physical Review E, vol. 66, no. 5, Article ID 056108, 7 pages, 2002.

[12] Y. Cho, H. Hajaiej, G. Hwang, and T. Ozawa, "On the cauchy problem of fractional schrödinger equation with hartree type nonlinearity," Funkcialaj Ekvacioj, vol. 56, no. 2, pp. 193-224, 2013.

[13] J. Dong and M. Xu, "Space-time fractional Schrödinger equation with time-independent potentials," Journal of Mathematical Analysis and Applications, vol. 344, no. 2, pp. 1005-1017, 2008.

[14] E. K. Lenzi, H. V. Ribeiro, M. A. F. dos Santos, R. Rossato, and R. S. Mendes, "Time dependent solutions for a fractional Schrödinger equation with delta potentials," Journal of Mathematical Physics, vol. 54, no. 8, Article ID 082107, 8 pages, 2013.

[15] A. D. Ionescu and F. Pusateri, "Nonlinear fractional Schrödinger equations in one dimension," Journal of Functional Analysis, vol. 266, no. 1, pp. 139-176, 2014.

[16] N. Hayashi and E. Kaikina, Nonlinear Theory of Pseudodifferential Equations on a Halfline, vol. 194 of North-Holland Mathematics Studies, Elsevier Science, Amsterdam, Netherlands, 2004.

[17] E. I. Kaikina, "Mixed initial-boundary value problem for intermediate long-wave equation," Journal of Mathematical Physics, vol. 53, no. 3, Article ID 033701, 22 pages, 2012.

[18] N. Hayashi and E. I. Kaikina, "Benjamin-Ono equation on a half-line," International Journal of Mathematics and Mathematical Sciences, vol. 2010, Article ID 714534, 38 pages, 2010.

[19] E. I. Kaikina, "Forced cubic Schrödinger equation with Robin boundary data: large-time asymptotics," Proceedings of the Royal Society of London, Series A: Mathematical, Physical and Engineering Sciences, vol. 469, no. 2159, Article ID 20130341, 16 pages, 2013.

[20] E. I. Kaikina, "A new unified approach to study fractional PDE equations on a half-line," Complex Variables and Elliptic Equations, vol. 58, no. 1, pp. 55-77, 2013. 


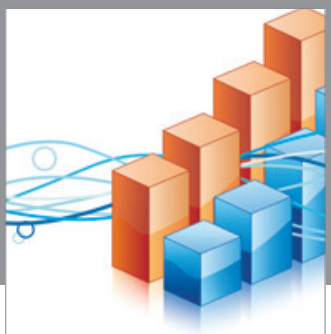

Advances in

Operations Research

vatem alat4

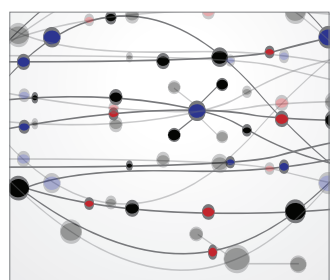

\section{The Scientific} World Journal
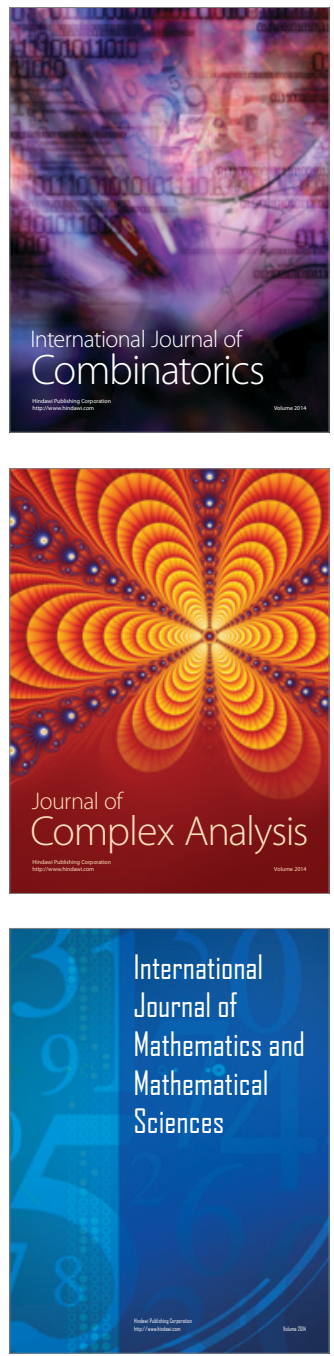
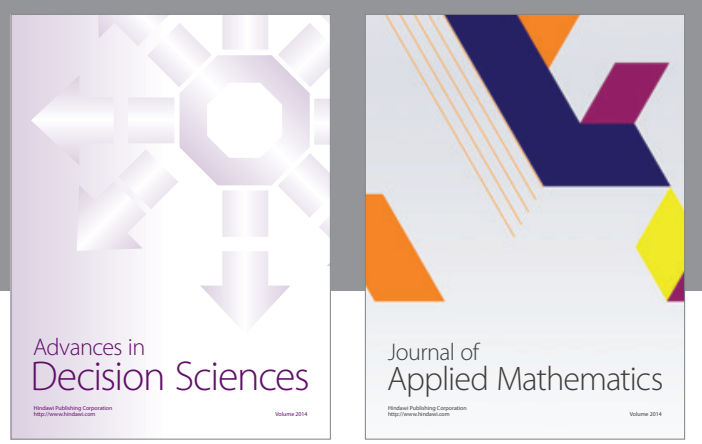

Algebra

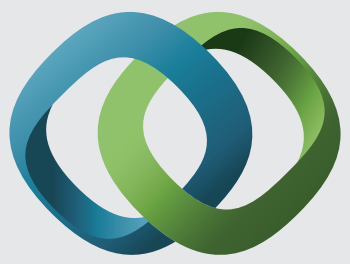

\section{Hindawi}

Submit your manuscripts at

http://www.hindawi.com
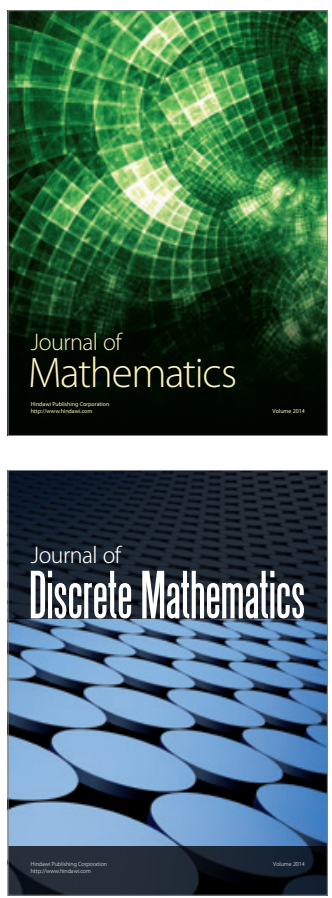

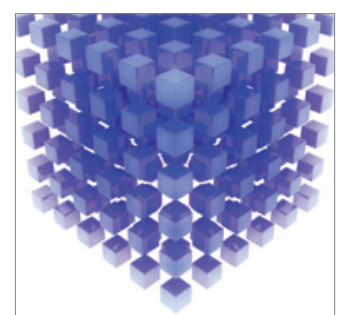

Mathematical Problems in Engineering
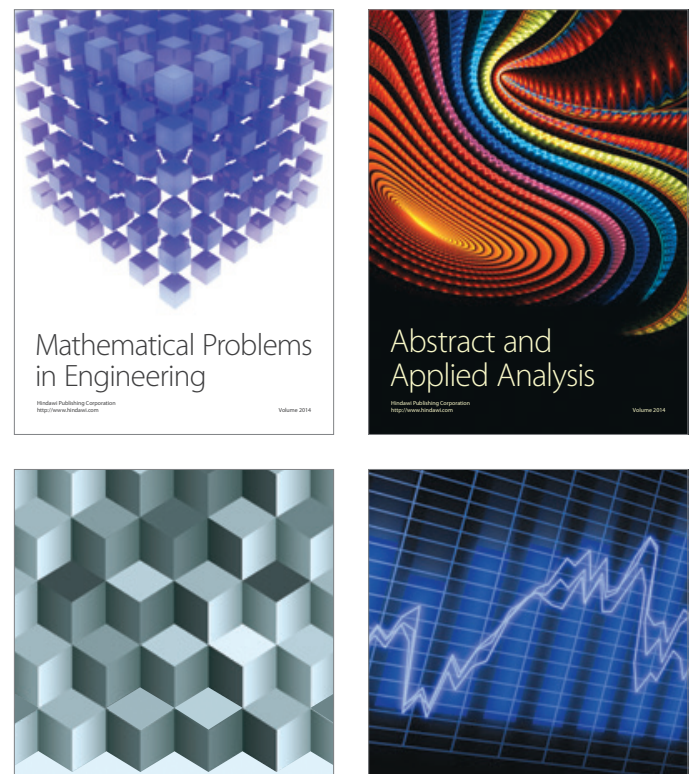

Journal of

Function Spaces

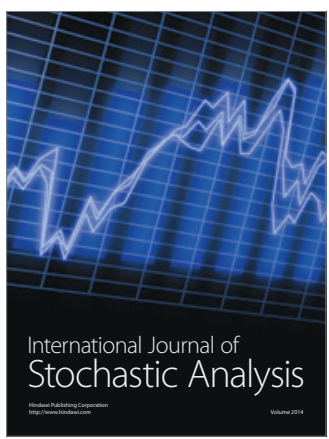

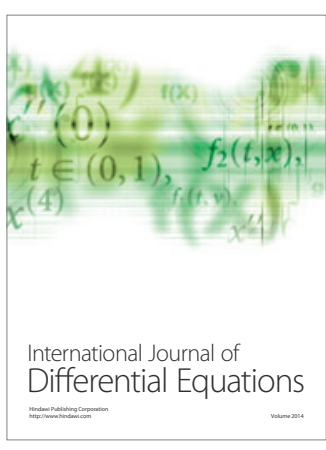
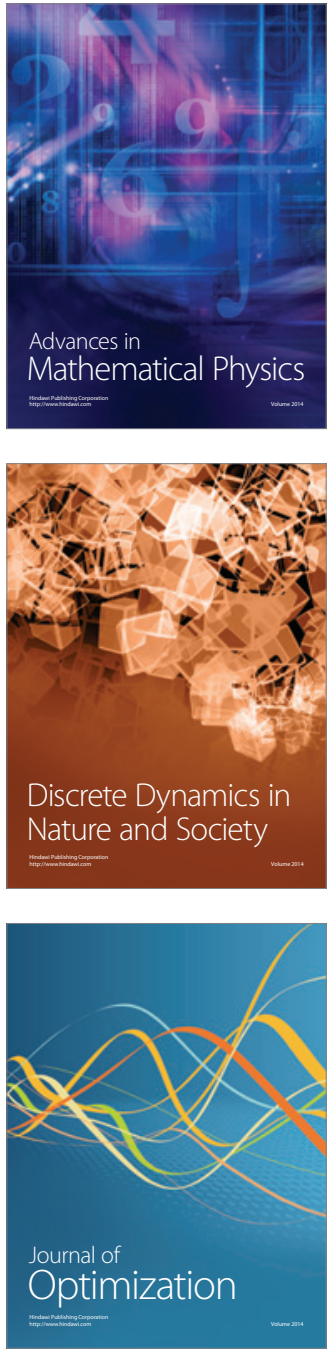